\title{
Frequency dependence of orthogonal polarisation modes in pulsars ${ }^{\star}$
}

\author{
J. M. Smits ${ }^{1}$, B. W. Stappers ${ }^{2,3}$, R. T. Edwards ${ }^{4}$, J. Kuijpers ${ }^{1}$, and R. Ramachandran ${ }^{5}$ \\ ${ }^{1}$ Department of Astrophysics, Radboud University Nijmegen, Nijmegen, The Netherlands \\ e-mail: R.Smits@science.ru.nl \\ 2 ASTRON, Dwingeloo, The Netherlands \\ 3 Astronomical Institute "Anton Pannekoek", Amsterdam, The Netherlands \\ 4 Australia Telescope National Facility, CSIRO, Australia \\ 5 Department of Astronomy, University of California, Berkeley, USA
}

Received 18 April 2005 / Accepted 21 November 2005

\section{ABSTRACT}

We have carried out a study of the orthogonal polarisation mode behaviour as a function of frequency of 18 pulsars, using average pulsar data from the European Pulsar Network (EPN). Assuming that the radiation consists of two 100\% polarised completely orthogonal superposed modes we separated these modes, resulting in average pulse profiles of each mode at multiple frequencies for each pulsar. Furthermore, we studied the frequency dependence of the relative intensity of these modes. We found in many pulsars that the average pulse profiles of the two modes differ in their dependence on frequency. In particular, we found that pulse components that are dominated by one mode tend to increase in intensity with increasing frequency with respect to the rest of the profile.

Key words. pulsar: general - polarization

\section{Introduction}

Single pulse studies of the position angle (PA) of linearly polarised radiation from a number of pulsars show that it is built up of two modes of polarisation, which are separated in angle by 90 degrees (e.g., Manchester et al. 1975; Stinebring et al. 1984; Rankin et al. 1988; Gil et al. 1991, 1992). It is believed that these orthogonally polarised modes (OPM) reflect the eigenmodes of the magneto-active plasma in the open magnetic field-lines above the pulsar polar cap. Three modes of wave propagation are allowed in this region, one of which is the ordinary sub-luminous mode, which cannot escape the pulsar magnetosphere due to Landau damping and is thus of no interest to the present work. The remaining two modes are the ordinary super-luminous mode (O-mode) which is polarised in the local plane defined by the external magnetic field and the wave-vector, and the extraordinary mode (X-mode), which is polarised perpendicular to this plane (Arons \& Barnard 1986; Petrova 2001). According to Barnard \& Arons (1986) refraction can separate the $\mathrm{X}$ - and O-modes beams, which have different indexes of refraction, by many beam-widths. The tendency for average profiles to have constant widths above

* Figures 4 to 24 are only available in electronic form at http://wwW.edpsciences.org a critical frequency is then explained by this separation occurring above a critical height (Sieber et al. 1975). Two conditions are required for this: (1) the radio emission mechanism has to be broadband in frequency over a narrow range of heights above the stellar surface and (2) the gradient in the plasma density has a transverse component to the radial direction (McKinnon 1997). The independent propagation of the two modes in the open flux zone can produce the abrupt orthogonal transitions in polarisation position angle that are commonly observed in studies of individual pulse polarisation (Manchester et al. 1975). The merging of the beams might also account for depolarisation of pulse average profiles with increasing radio frequency (McKinnon 1997; von Hoensbroech \& Xilouris 1997). Alternatively, the OPM transitions might not be due to refraction, but due to switching between significant and insignificant conversion of O-mode into X-mode (Petrova 2001).

One of the most important questions about OPM is whether the polarisation modes are disjoint or superposed. For disjoint modes the polarisation of the observed radiation is given by either one of the two modes at each point in time. Superposed modes occur simultaneously and the polarisation of the observed electromagnetic radiation is given by the vectorial addition of the Stokes parameters $Q, U$ and $V$ of both modes. 
Cordes et al. (1978), who first posed this question based on the polarisation from PSR B2020+28, assumed the modes to be disjoint and stated three arguments for this assumption: (1) the degree of polarisation is fairly steady from one pulse to the next, (2) if the modes are superposed, one might expect occasional sign changes in the complex value $Q+\mathrm{i} U$ when the linear polarisation is low, due to random fluctuations in the relative strength of the two modes and (3) the instantaneous mode of polarisation seems correlated with the total intensity, which is consistent with disjoint occurrence of the modes. However, Stinebring et al. (1984) found that the modes appear to be superposed by studying sensitive polarisation observations of a number of pulsars. It was found that average values of the fractional linear polarisation were small at longitudes where both polarisation modes occurred with nearly equal frequency. The same effect was later found in the instantaneous values of the fractional linear polarisation in single pulse observations of PSR B2020+28 (McKinnon \& Stinebring 1998). This phenomenon is present on timescales of hundreds of microseconds to hours, which is what one would expect for superposed OPM. For disjoint modes one would expect the fractional polarisation to be either high or low, depending on the fractional polarisation of the active mode.

Assuming that the polarisation modes are superposed, that they are completely polarised and completely orthogonal, the average intensity of the individual polarisation modes as a function of longitude can be determined from the Stokes parameters of the average pulsar signal (McKinnon \& Stinebring 2000). In this paper we make these assumptions and determine the individual polarisation profiles of 18 pulsars as a function of both longitude and frequency (using data from the EPN database). In Sect. 2 we give a description of our analysis, the results of which are shown in Sect. 3. In Sect. 4 we discuss the results and finally we give our conclusions in Sect. 5.

\section{Method}

McKinnon and Stinebring have developed a method to separate the modes of electromagnetic radiation that is built up out of two $100 \%$ polarised completely orthogonal modes that are superposed (McKinnon \& Stinebring 2000). This method is illustrated by means of vectors in Fig. 1. Each pulse longitude will have a value for Stokes $Q, U$ and $V$, which can be expressed by a polarisation vector in $Q-U-V$-space (Poincaré space). The intensity corresponds to the radius of the sphere. Polarisation vectors lying on the sphere indicate full polarisation, whereas vectors lying within the sphere indicate partial polarisation. The fraction of polarisation is given by the length of the polarisation vector divided by the radius of the sphere (the intensity). Furthermore, two vectors with orthogonal polarisation will be antiparallel. The method first fixes the orientation of the polarisation vectors to lie in the $Q-V$ plane, shown in Fig. 1, by rotating the vector around the $V$ axis. In this figure, the average intensity of the observed radiation is given by the circle with radius $I_{\mathrm{e}}$, the observed polarisation vector is given by $P_{\mathrm{e}}$ and the underlying polarisation of the orthogonal modes are given by $P_{1}$ and $P_{2}$. There are now two relationships between the observed intensity and polarisation vector $\left(I_{\mathrm{e}}\right.$ and $\left.P_{\mathrm{e}}\right)$ and the

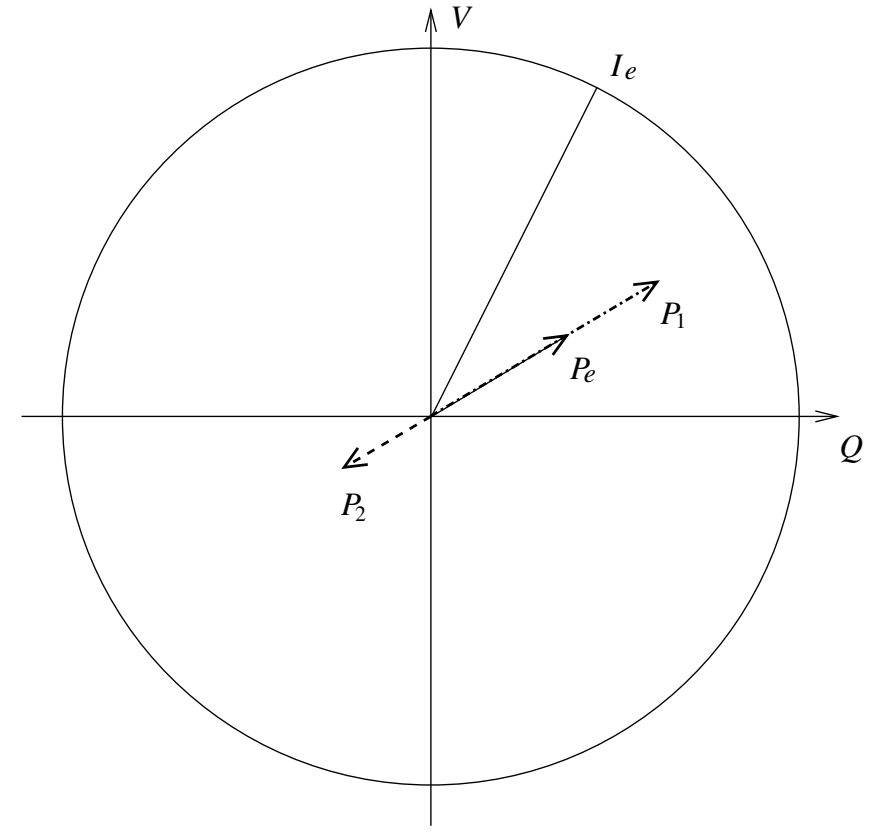

Fig. 1. Poincaré circle that illustrates the method used to determine the intensity and polarisation of two orthogonally polarised modes from the observed radiation. $P_{1}$ and $P_{2}$ represent the antiparallel polarisation vectors of both modes. The radius of the circle, $I_{\mathrm{e}}$, represents the total intensity of the radiation, which is equal to the sum of the lengths of vectors $P_{1}$ and $P_{2}$, because the polarisation modes are superposed incoherently. $P_{\mathrm{e}}$ represents the polarisation of the electromagnetic radiation which is equal to the vectorial sum of $P_{1}$ and $P_{2}$. Note that the polarisation vector of the observed radiation has been rotated around the $V$ axis to lie in the $Q-V$ plane.

polarisation vectors of the orthogonal modes $\left(P_{1}\right.$ and $\left.P_{2}\right)$. First, $I_{\mathrm{e}}$ is equal to the sum of the lengths of $P_{1}$ and $P_{2}$ and second, $P_{\mathrm{e}}$ is equal to the vectorial sum of $P_{1}$ and $P_{2}$. With these relationships the two orthogonal polarisation vectors can easily be determined from the Stokes parameters.

We apply this method to 18 pulsars for which polarimetric average pulse profiles are available in the EPN database. Given the assumptions that the radiation is built out of two superposed orthogonal modes that are $100 \%$ polarised, the separation is completely correct. Because the superposition is linear, separation of the average polarisation profile at each longitude will result in the means of the individual modes at that longitude. Because of this, we do not see the polarimetric behavior of single pulses, nor any polarimetric behavior on small timescales. If, for example, the separation from the average polarisation profile at a fixed longitude would reveal both modes to be of equal strength, then one solution might be that at this longitude all single pulses are built out of the superposition of two modes with equal strength. Yet it might also be the case that at this longitude one half of the single pulses would be built out of just one of the orthogonal modes and the rest of the single pulses would be built out of just the other mode. The data from the EPN database consists of time-averaged data at multiple frequencies. Of these pulsars, 11 are known from previous studies to show OPM. It should be noted that this method gives separated intensities corresponding to both modes at each 
longitude, but there is no direct knowledge as to determine which value belongs to which mode. We therefore have to be cautious when making a longitude plot of the intensity of both modes as there are always two possibilities when connecting intensity points from one longitude to the next. Generally, it is safe to assign values with the highest intensity to one mode and values with the lowest intensity to the other. When these values lie very close to each other, the assignment of value to modes is made on the basis of visual inspection for changes in modal dominance as evidenced by sharp jumps of nearly $90^{\circ}$ in the PA. In some cases the intensity points are connected by finding a smooth line through the values. We have defined the polarisation mode with the highest integrated intensity at the lowest available frequency as the strongest mode. We thus produce the intensity of the separated modes, as well as the PA and ellipticity angle $\left(\frac{1}{2} \operatorname{atan}\left(V / \sqrt{Q^{2}+U^{2}}\right)\right.$ as a function of frequency.

Furthermore, we have calculated the ratio of the longitudeaveraged intensity of both modes at each frequency. For pulsars with two pulse components this has been done for both components separately and for both components added together. The error on the average intensity was calculated as $\sigma_{I}=\sqrt{N} \cdot \sigma$, where $N$ is the number of bins of the profile and $\sigma$ is the noise level, given by the standard deviation of the noise as estimated from data in the off-pulse region of the profile. For 14 pulsars, the pulse profiles at all frequencies were aligned by visual comparison of the profiles with the corresponding plots from Kuzmin et al. (1998). When a profile at a particular frequency had no direct comparison, the alignment was estimated from the frequency development from the plots from Kuzmin et al. (1998). Pulsars B0144+59, B1039-19, B1800-21 and B1944+17 were not listed in this paper and were aligned by comparing pulse components and characteristics in the polarisation from frequency to frequency. We can therefore not guarantee that these pulsars are aligned correctly.

\section{Results}

If we assume that emission altitude is inversely related to the frequency of emission (radius to frequency mapping) and that the magnetic field-lines are dipolar shaped at the emission height, observing at different frequencies has two implications: (1) radiation at high frequency arises lower in the pulsar magnetosphere than radiation at low frequency and (2) radiation observed at high frequency is emitted at field-lines that intersect the surface further away from the magnetic axis than radiation observed at the same pulsar phase at low frequency (see Fig. 2). When studying the average profiles at multiple frequencies, this should be kept in mind.

Figures 4 through 21 show the results of the mode separation at all frequencies for all pulsars. The left columns show the average total intensity (solid line) and the average intensity of both modes (dotted and dashed lines), the middle columns show the PA of the average Stokes parameters and the right columns show the ellipticity angle at all frequencies. The PA and ellipticity angle at pulse longitudes where the polarised intensity is less than twice the rms noise are not shown. Figures 22 through 24 show the frequency

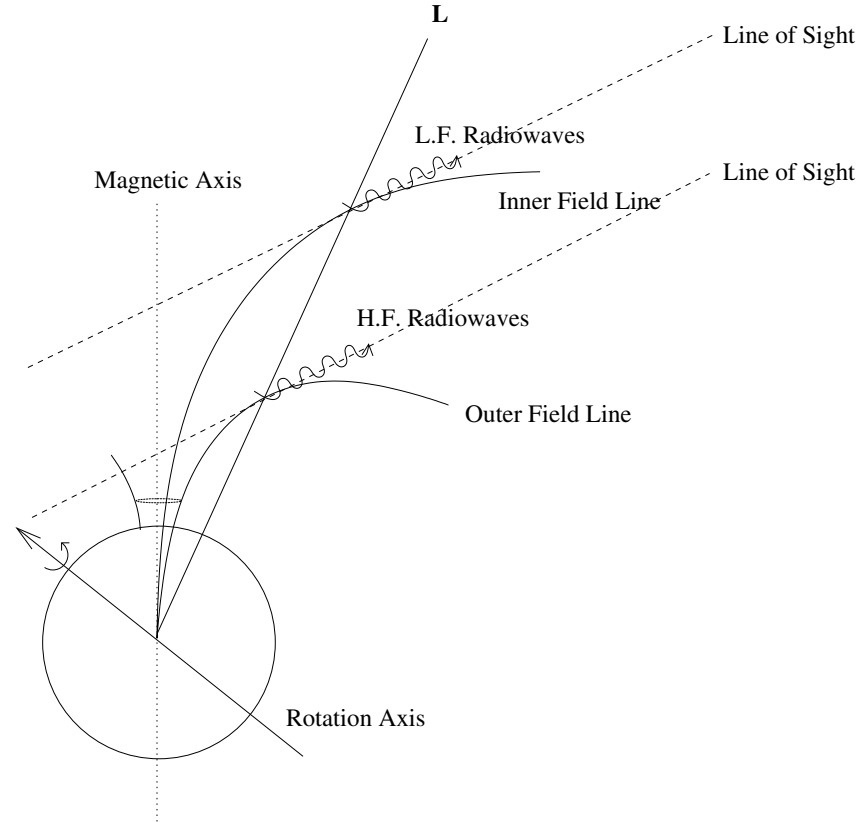

Fig. 2. Schematics of two field lines from which radio waves of two different frequencies are observed. $\mathrm{L}$ is the line that connects the locations where the dipolar field lines are directed towards the observer. Due to radius-to-frequency mapping, low frequency radio waves are emitted higher in the magnetosphere than high frequency radio waves. As the image shows, the low frequency radio waves that can be observed are emitted on field lines that intersect the surface closer to the magnetic axis than the field lines that emit the observed high frequency radio waves.

development of the ratio of the average intensities of both modes, plotted on a log-log scale, for all pulsars. The straight line through the points was determined by the least squares fitting technique. Whenever these points show little deviation from the straight line fit, the related power law index is shown in Table 1. As there is no preferred mode, the values are always taken to be positive. The point-style indicates the reference corresponding to the source of the data in the EPN, which is listed below Fig. 24. The polarisation of the $1.41 \mathrm{GHz}$ data from von Hoensbroech \& Xilouris (1997) was frequently found to differ from other $1.41 \mathrm{GHz}$ EPN data and from the development of the polarisation as a function of frequency. Thus these data were not included in the analysis.

Let us now consider each pulsar in detail. For some pulsars we will refer to the classification by von Hoensbroech et al. (1998), who have classified a number of pulsars into four groups, named after one of the pulsars in that group. The first group of pulsars is exemplified by type $0355+54$, for which one component is highly polarised, whereas the rest of the profile has little polarisation. The component that is highly polarised has a flatter spectral behaviour than the rest of the profile and therefore dominates at higher frequency. The second group consists of $0525+21$-like pulsars, which, in the classification system from Rankin (1983), are all examples of conal doubles. They have two moderately linearly polarised components, the PA follows the rotating vector model (RVM) of Radhakrishnan \& Cooke (1969) nearly perfectly and there 
is usually a core-type bridge between the components. The third group consists of 1800-21-like pulsars, which are young, have a very high spin down power, $\dot{E}$, a relatively flat radio spectrum behaviour, detectable $\mathrm{X}$-ray emission and radio emission over a wide fraction of the pulse period. They're also nearly fully polarised, linearly and circularly and do not show the commonly observed effect of depolarisation towards high frequencies. The final group consists of 0144+59-like pulsars, which show an unusual polarisation behaviour. Their degree of circular polarisation increases strongly with frequency.

\section{$B 0144+59$}

Pulsar B0144+59 defines the class of 0144+59-like pulsars, as described above. Figure 4 shows that at all frequencies except $4.85 \mathrm{GHz}$ there is a mode-switch around $-2^{\circ}$, which is associated with a jump in the PA. At $0.4 \mathrm{GHz}$ the first component can be associated with one mode, whereas the second and third components seem to be associated with the other mode. Although the intensity fluctuations around $2^{\circ}$, which suggest a second and third component, are not significant and might be just one component. The association of the modes with components is also valid at higher frequencies, even when the first component becomes weaker and almost disappears at $4.85 \mathrm{GHz}$. At this frequency the remaining component is almost completely dominated by one mode. Also, the slope of the position angle traverse increases. This pulsar shows an example of a component (left component) being dominated by a single mode and slowly disappearing with increasing frequency. It would be interesting to see whether this trend continues towards even lower frequencies. Unfortunately, such data was not at our disposal. The frequency development of these modes is nicely fit by a power law.

\section{B0355+54}

Pulsar B0355+54 defines the class of 0355+54-like pulsars, as mentioned above. Figure 5 shows that the leading component consists of mainly one mode (the stronger mode) and the second component is dominated by the other mode. At all frequencies, except perhaps for $10.55 \mathrm{GHz}$, the PA jumps and the ellipticity angle switches sign at the same longitude where the modes change dominance. At low frequency the dominance of the weaker mode is only a few degrees in longitude, but becomes broader with increasing frequency. This mode can be associated with the second component, but the stronger mode is present there as well. The first component rises in intensity at higher frequencies, relative to the intensity of the first component, which contains only one mode. This behavior has already been noted by von Hoensbroech et al. (1998). The PA sweep of the left component is straight and has a small slope, which slightly increases with increasing frequency. The right component shows the $90^{\circ}$ jump, after which the sweep remains straight for a few degrees (the number of degrees depending on frequency) and then has a smaller jump, followed by a curved sweep. The second jump in the PA can be associated with a second change in dominance of the two modes. However, at high frequencies this is not clear. It is also not clear whether the curve at the trailing edge of the PA is the natural PA sweep of one of the modes, or that the curve is the result of a (nonorthogonal) superposition of both modes. The frequency development of the modes is nicely fit by a power law, except for a jump at $1.642 \mathrm{GHz}$.

\section{$B 0450+55$}

Pulsar B0450+55 falls into the same group of pulsars as B0355+54 (von Hoensbroech et al. 1998). Figure 6 shows that at a frequency of $0.910 \mathrm{GHz}$ the PA jumps $90^{\circ}$ twice around $0^{\circ}$ longitude, indicating a brief mode-change which is visible in the average profile of the modes. The first jump shows a gradual change. The ellipticity angle switches sign at the same longitude in accordance with the mode-change. At lower frequencies the PA also jumps twice at the same longitudes, but this does not show up in the average profile of the modes. These jumps are less than $90^{\circ}$ and also show a gradual change. It is interesting that at $0.404 \mathrm{GHz}$ the first jump is upwards while at $0.610 \mathrm{GHz}$ the first jump is downwards, while the rest of the PA sweep is very alike. The first component of the average intensity is strongly dominated by one mode and the intensity of this component increases with frequency relative to the second component, in which both modes are present and nearly equally strong. Because it is dominated by one mode, Fig. 22 does not show the frequency development of the modes in the left component. For both the right component and the entire profile, the frequency development of the modes do not seem to be fit by a power law.

\section{$B 0525+21$}

Pulsar B0525+21 defines the class of B0525+21-like pulsars, as described above. Figure 7 shows that both modes are active in both components. At high frequency, there is strong depolarisation in both components, which is represented by the weaker mode becoming stronger at higher frequency. The modes in both components behave very similarly, although the ratio between integrated intensity of the stronger and weaker mode is higher in the right component. The intensity of this component increases with frequency, relative to the left component. The PA sweep at $4.85 \mathrm{GHz}$ shows a $90^{\circ}$ phase jump, suggesting a mode-switch somewhere in the bridge between the two components. However, the frequency development of the PA sweep clarifies that this jump is actually the result of the normal RVM. The ellipticity angle gives little information about mode-switching, because of the low circular polarisation. This leaves us with no indication of mode-switching in this pulsar. The frequency development of the modes are nicely fit by a power law in both components.

\section{$B 0809+74$}

The polarisation of pulsar B0809+74 has been well studied in the past. Recently, Rankin et al. (2004) has shown that the two orthogonal modes of polarisation are the 
primary reason for a variation in the longitudinal separation between the drifting subpulses of pulsar B0809+74. Furthermore, Edwards (2004) has confirmed that the polarisation fluctuations are the result of two orthogonally polarised modes, although an apparantly randomly polarised component needs to be superposed. The pulsar has two components. The first component arises with increasing frequency and becomes visible around $0.925 \mathrm{GHz}$ and higher and is almost completely dominated by the stronger mode (see Fig. 8). In the second component both modes are almost equally strong. At frequencies of 0.606 and $1.408 \mathrm{GHz}$, the PA jumps by approximately $60^{\circ}$ and $80^{\circ}$, respectively. This does not seem to be associated with a mode-change. There is no clear frequency development of the modes.

\section{$B 0823+26$}

Pulsar B0823+26 has only one component, in which both modes are active (see Fig. 9). Both the profile and the relative intensity of the modes do not change very much over frequency. Around the middle of the profile there is a small jump in the PA sweep, which increases in size with increasing frequency. At the same longitude as this jump the modes are almost of equal strength. Also, the weaker mode becomes stronger with respect to the stronger mode at higher frequency. The frequency development of the modes are nicely fit by a power law.

\section{B0834+06}

Pulsar B0834+06 consists of two components with a high bridge connecting them. From studies of individual-pulse polarisation observations it has been concluded that both modes are equally strong in the two components, but only one mode dominates across the bridge (Stinebring et al. 1984). However, Fig. 10 shows that the difference in average intensity between the modes is small across the bridge as well. The PA sweep at $0.408 \mathrm{GHz}$ and $1.408 \mathrm{GHz}$ are very similar, however, the PA sweep at $0.610 \mathrm{GHz}$ has a drop in the center of the profile. Except for one point with a large error, the frequency development of the modes can be fit by a power law. But since there are only four points in total, this is not very significant.

\section{B0919+06}

The average profile of pulsar B0919+06 changes greatly over frequency. At low frequency there seem to be two components visible, as well as a slope at the leading edge. With increasing frequency the two components merge into one and the slope becomes smaller and even disappears at $4.85 \mathrm{GHz}$. The profile is dominated by one mode, with the exception of the slope and part of the left component, in which both modes are almost equally strong (see Fig. 11). The weaker mode moves to the right with respect to the stronger mode, at rising frequency. This results in the extra component at low frequency $(0.408 \mathrm{GHz})$, associated with the weaker mode, to disappear towards higher frequencies. There is no indication of modeswitching. The frequency development of the modes seem to be fit well by a power law, with the exception of the datapoint at $4.85 \mathrm{GHz}$.

\section{$B 0950+08$}

Pulsar B0950+08 is remarkably similar to pulsar B0919+06. They both have one dominant component a weak second component at low frequencies and a slope at the leading edge. However, the polarisation modes of pulsar B0950+08 are almost equally strong over the entire frequency range and do not show any drift (see Fig. 12). There is no evidence of a modeswitch in the average profile. The PA does rise and fall where the modes have nearly equal power, but only by $70^{\circ}$. The rise in the PA becomes broader with increasing frequency and at 1.642 and $4.850 \mathrm{GHz}$, the PA has changed significantly. At $1.642 \mathrm{GHz}$ it is clear that the PA drops instead of rises and by a smaller amount than at lower frequency. The ellipticity angle falls and rises at the same longitude at which the PA changes. This dip also becomes broader towards higher frequency. There does not seem to be a clear frequency development of the modes.

\section{B1039-19}

Pulsar B1039-19 is B0525+21-like. Figure 13 shows that both modes are active in both components and that the PA does indeed follow the RVM. At higher frequency, the weaker mode becomes stronger. At low frequency the PA sweep shows a typical RVM curve, but towards higher frequency the sweep starts with a rise and then drops in a straight line. For the first components, the frequency development of the modes seems to be fit by a power law. For the second component the fit is not so good, but the same trend is visible.

\section{$B 1133+16$}

This pulsar has two components. In the left component both modes are almost equally strong (see Fig. 14). The right component is dominated by one mode at low frequencies, but the modes become almost equally strong at higher frequencies. This behavior is the opposite of what would be expected by comparing the results on the polarisation of $\mathrm{B} 1133+16$ from Backer \& Rankin (1980) (hereafter BR) with the results from Stinebring et al. (1984) (hereafter SCRWB). It can be seen there, that at low frequency the right component shows OPM (Fig. 9 in BR), which becomes less visible at higher frequency (Fig. 17 in SCRWB). However, OPM are expected to occur when both modes are about equal in strength which, according to our results, is at high frequency rather than at low frequency. This could indicate that the method for separating the modes is not succesful in this case. However, we must keep in mind that we are only showing average profiles. If the (refracted) path of one of the polarisation modes in the magnetoactive plasma can vary in time, then the strength of the polarisation modes can briefly change, possibly making OPM visible in single pulse studies. At $1.408 \mathrm{GHz}$ the PA jumps upward by $90^{\circ}$ and jumps downward again one degree later, which results in 
a mode-change, as can be seen in the plot of the average intensity of the modes. At $1.642 \mathrm{GHz}$ this jump is still present, but has become more gradual, is less than $90^{\circ}$ and does not result in a mode-change. At 1.71 and $4.85 \mathrm{GHz}$ these jumps are also present, but are first downward, then upward. At $4.85 \mathrm{GHz}$ the average intensity profile of the modes show a brief modechange. The modes in the left component seems to have a clear frequency development, whereas the modes in the right component do not.

\section{$B 1237+25$}

Pulsar B1237+25 is known for its profile "mode-switching" (which means that it has two different average profiles, called "modes", which are not to be confused with the orthogonal polarisation modes) (Bartel et al. 1982). By comparing the EPN profiles of pulsar B1237+25 with those in (Bartel et al. 1982), we conclude that the abnormal mode can only be faintly present in the average pulse profiles. There are many components in this pulsar, which are all dominated by the stronger mode (see Fig. 15). While the weak component at a longitude of $-3^{\circ}$ can be associated almost completely with the stronger mode, the weak component at a longitude of 1.5 can be associated with the weaker mode. These associations are strongest at low frequencies. At higher frequency, the weaker mode becomes stronger with respect to the stronger mode. According to $\mathrm{BR}$, this pulsar shows OPM in the two main components at a frequency of $0.430 \mathrm{GHz}$, which, as in the right component of pulsar B1133+16, is not consistent with the result of one dominant mode. At almost all frequencies, the position angle rises and falls along the bridge between the two dominant components. At this same longitude, there is a lot of circular polarisation. This discontinuity in the PA is not associated with OPM (Stinebring et al. 1984), but it does occur at a longitude where the weaker mode becomes stronger with respect to the stronger mode. Both the frequency development of the intensity of the modes in the left and right half of the profile follows a power law, but the modes in the right half have a very low power law index.

\section{B1737-30}

Pulsar B1737-30 has only one component and falls into the same group of pulsars as B0144+59 (von Hoensbroech et al. 1998). At a frequency of $1.408 \mathrm{GHz}$ and higher, the component is almost completely dominated by one polarisation mode. At the trailing edge of the component the amount of circular polarisation increases. This behaviour is intrinsic to the modes themselves, as there is mainly one mode active throughout the profiles. The frequency development of the intensity of the modes seem to follow a power law.

\section{B1800-21}

Pulsar B1800-21 defines the class of 1800-21-like pulsars, as described above. At low frequency this pulsar has up to 4 components, but at high frequency this pulsar has only two components, both dominated by the stronger mode (see Fig. 17). The first component arises at a frequency of $1.408 \mathrm{GHz}$. At 1.408, 1.56, 1.642 and $4.852 \mathrm{GHz}$ there is a gap in the PA due to the low signal in the bridge between the two main components. The PA at $4.85 \mathrm{GHz}$ shows the PA sweep over this bridge and clarifies that the stronger mode is dominant in both components. There is, however, a brief mode-switch in the bridge between the two main components at $1.408 \mathrm{GHz}$ and another one at the trailing tail of the second component. At frequencies below $4.85 \mathrm{GHz}$ the peak of the weaker mode is separated by approximately $15^{\circ}$ $(\approx 5.6 \mathrm{~ms})$ from the peak of the stronger mode. At a frequency of $4.85 \mathrm{GHz}$, the weaker mode has almost completely disappeared. There is no clear frequency development of the intensity of the modes, but it is clear that the weaker mode becomes weaker with increasing frequency.

\section{$B 1929+10$}

The profile of pulsar B1929+10 changes greatly over frequency, but at all frequencies there is only one dominant mode (see Fig. 18). There is OPM at the trailing part of the main component (Stinebring et al. 1984), which coincides with the rise of the weaker component. At $4.85 \mathrm{GHz}$ the position angle suggests that there is a mode-switch in the leading edge of the profile. Due to the low intensity it is difficult to confirm this with the intensity plots. It is interesting that the weaker mode retains its shape and position, whereas the components of the stronger mode change greatly in shape and position. This is especially evident at $4.85 \mathrm{GHz}$ where one might naively expect the two components in the average intensity profile to lie at the same longitude as the second and third components of the 1.71-GHz profile, rather than to coincide with the first and second components. However, using the alignment from Kuzmin et al. (1998) the weaker mode does not change in longitude over frequency, which suggests the alignment to be correct. There is no clear frequency development of the intensity of the modes, but it seems that the weaker mode becomes stronger with increasing frequency.

\section{B1944+17}

Pulsar B1944+17 switches modes at the leading and trailing edge of the pulse at 0.610 and $1.408 \mathrm{GHz}$ (see Fig. 19). Overall, the weaker mode has different intensity at different frequencies. It is roughly $1 / 4$ of the dominant mode at frequencies of 0.410 and $0.925 \mathrm{GHz}$ and about $1 / 2$ of the strength of the dominant mode at frequencies of $0.61,1.408$ and $4.85 \mathrm{GHz}$. However, there is no clear frequency development of the intensity of the modes.

\section{$B 2016+28$}

The profile of pulsar B2016+28 has two components, which become less distinct towards higher frequency (see also (Izvekova et al. 1993)). Figure 20 shows that these components can be associated with the two different modes. At low 
frequency, the weaker mode consists of two components and of only one component at higher frequency. The peak of the modes move towards each other with increasing frequency and become of almost equal strength. At frequencies higher than $1.408 \mathrm{GHz}$, the shapes of the two modes are almost similar. Due to this behavior, the left part of the profile is dominated by one mode at low frequencies while it is composed of two equally strong modes at high frequency. This pulsar is known for its mode-changing (Stinebring et al. 1984; Arons \& Barnard 1986). This can be seen at a frequency of $0.408 \mathrm{GHz}$, where the modes switch dominance. At the same longitude the PA jumps by approximately $90^{\circ}$, however the PA at $1.408 \mathrm{GHz}$ has a similar behavior and does not seem to be associated with a mode-change. This suggests that the jump in the PA at $0.408 \mathrm{GHz}$ and $1.408 \mathrm{GHz}$ are due to the RVM and respresent the PA sweep of the dominant mode. Moreover, at 0.610 and $0.925 \mathrm{GHz}$ the PA jumps downwards and does not seem to be associated with a mode-change. It therefore remains unclear what the true PA sweep of this pulsar is. There does not seem to be a frequency development of the intensity of the modes.

\section{$B 2020+28$}

Pulsar B2020+28 is known for its abrupt mode-change (Cordes et al. 1978; Stinebring et al. 1984; Arons \& Barnard 1986). This can be seen in the PA at all frequencies (see Fig. 21). This pulsar also shows a large drift of the modes with respect to each other. Figure 3 shows the difference in longitude between the peaks of the two modes as a function of frequency. (The large errors are because of the uncertainty in the position of the peak of each mode.) The line through the points is a fit of the function $a \log (f)+b$ of the points, where $f$ is the frequency in $\mathrm{GHz}$ and $a$ and $b$ are fit parameters. The values of these fit parameters are $a=0.56 \pm 0.11$ and $b=8.54 \pm 0.13$. There does not seem to be a frequency development of the intensity of the modes.

\section{Discussion}

Data from the EPN is very suitable to apply the modeseparation technique for superposed modes, as prescribed by McKinnon \& Stinebring (2000). This makes a frequency analysis of OPM possible. Applying this technique to EPN data has three drawbacks (1) the pulse-profiles at different frequencies are not aligned, (2) the information on the observed pulsar flux is seldom available and (3) the data only show average profiles, which might not represent the behavior of single pulses. Still, the results described here, give a clear picture of how the average properties of the two modes behave as a function of frequency.

In Table 1 we summarise the results of the analysis of the orthogonally polarised modes of the 18 pulsars, as well as some of the known parameters. $w_{50}$ and $w_{10}$ are the pulse widths at $50 \%$ and $10 \%$ of the peak flux, respectively, $S_{400}$ and $S_{1400}$ are the flux densities at 0.4 and $1.4 \mathrm{GHz}$ respectively and $\dot{E}$ is the spin-down luminosity, given by $-I \Omega \dot{\Omega}$, where $I$ is the pulsar's moment of inertia for which we take $10^{45} \mathrm{~g} \mathrm{~cm}^{2}$ and $\Omega$ and $\dot{\Omega}$ are the rotation frequency and the time derivative of

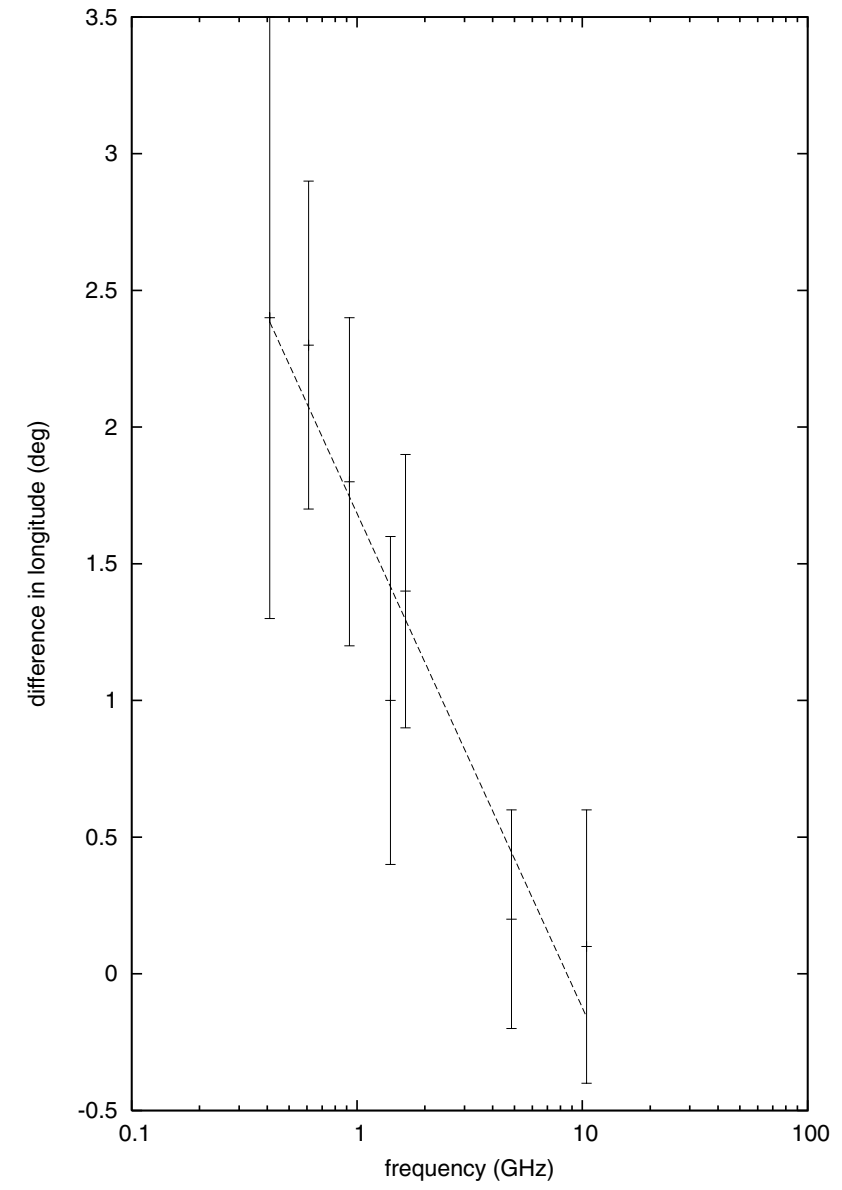

Fig. 3. Separation in longitude between the peaks of the average intensity of the two orthogonally polarised modes of PSR B2020+28 as a function of frequency. The dashed line is the best straight line fit through the points.

the rotation frequency of the pulsar, respectively. Values for the period, pulse widths and flux densities are from Taylor et al. (1993). Type and $\alpha$ and $\beta$ parameters are from Rankin (1993), except for pulsars B0144+59 and B1800-21 for which we have determined the type ourselves from the polarisation characteristics. The types are based on the classification system from Rankin (1983) which assumes a core radiation zone, surrounded by a conal radiation zone. They have the following meaning. $S_{\mathrm{d}}$ is a single component profile; the line of sight grazes the outer cone of the polar cap. D is a double component profile; the line of sight cuts through the outer cone twice, but misses the core component. $T$ is a triple component profile; the line of sight cuts through the outer cone twice as well as the core. $S_{\mathrm{t}}$ is a single component profile; in this case the outer cone is missing and the line of sight only cuts through the core. Finally there are two types that, in this view, can only be explained by two conal radiation zones around the core component; they are $\mathrm{M}$, which is a multiple component profile (containing usually 5 components) and $T_{\mathrm{c}}$, which is a triple component profile, which consist of three conal components.

Let us now consider some of the observed phenomena. 
Table 1. List of characteristics of the orthogonally polarised modes of analysed pulsars.

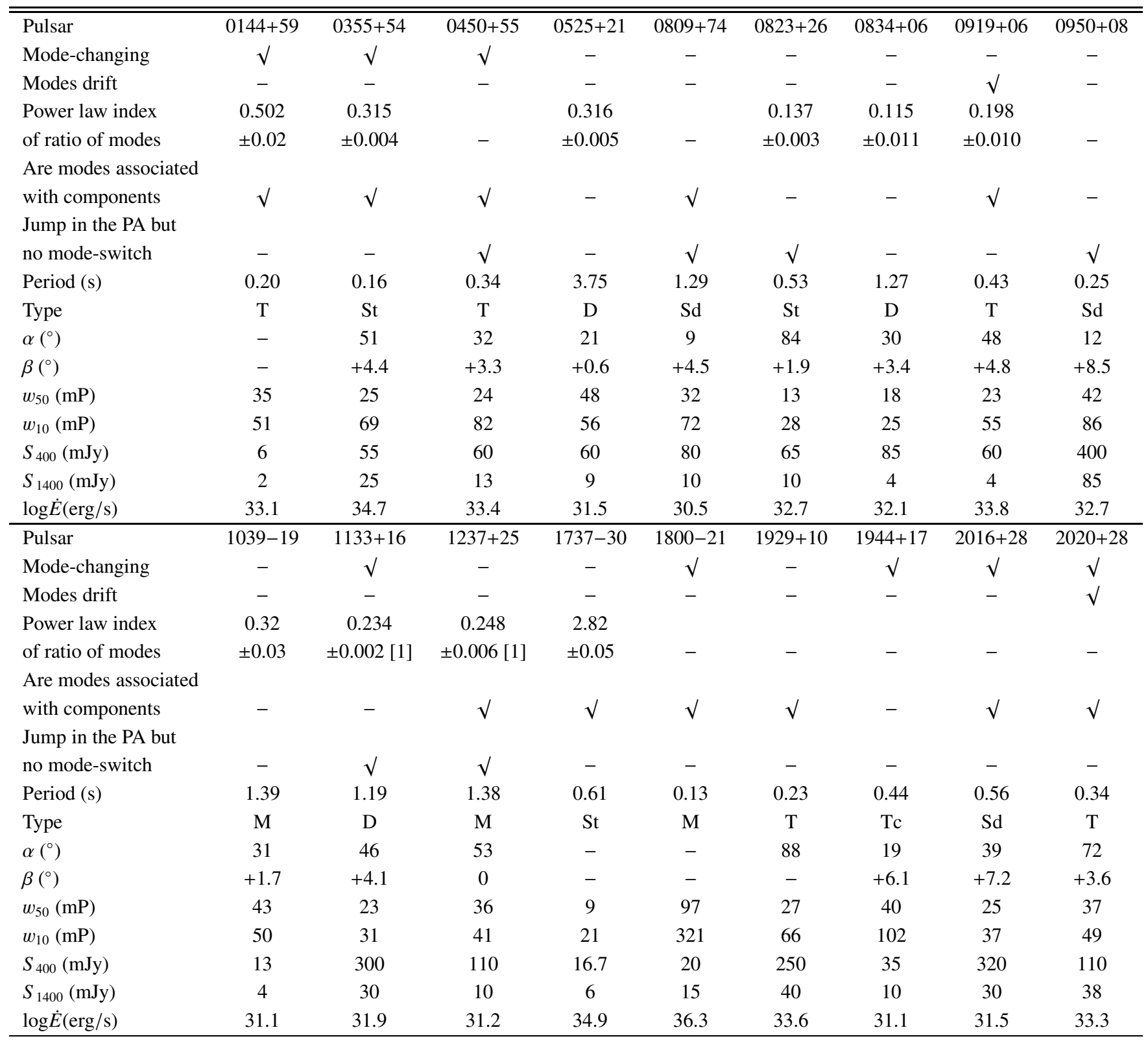

[1] Left component only.

\section{Mode-changing}

In almost half of the pulsars, there is a jump of $90^{\circ}$ in the PA, which can always be associated with a change in the dominance of the modes. (See first row of Table 1.) This can be during a fraction of the pulse period, as in pulsar B1133+16, or for most of the remaining profile, as in pulsar B0144+59. In the interpretation of the two orthogonal modes corresponding to the ordinary and extraordinary modes (Barnard \& Arons 1986; Petrova 2001), mode-changing is likely to be a result of the spatial separation of these modes due to refraction. Figures 4 through 21 show that mode-changing can occur at any frequency. A comparison between our intensity profiles of the polarisarion modes of pulsars B1133+16 and B1237+25 with the results from a single pulse study (Backer \& Rankin 1980) suggests that OPM can become visible in single pulse studies even when there is one dominant mode thoughout the average profile. Figures 4 through 21 show that in many pulsars there is a great change in the average profile of the two polarisation modes over frequency, suggesting in contrast to common belief, that refraction can take place at all heights of emission.

\section{Mode-drifting}

In two cases, the longitudinal position of one of the modes relative to the other can be seen to change as a function of frequency (see Table 1). For pulsar B2020+28, the longitudinal separation between the modes as a function of frequency is shown (see Fig. 3). Assuming radius to frequency mapping, this drift of one of the modes can be interpreted as a change 
of refraction with emission altitude. For pulsar B0919+06 the mode drift is less pronounced and whereas the drifting mode of pulsar B2020+28 moves away from the center with increasing frequency, the drifting mode of pulsar B0919+06 moves towards the center with increasing frequency. The relative drift of the two modes might be correlated to other observable features, which have to be common features of both pulsars. Table 1 shows the common features of these two pulsars. They both have a period close to $0.4 \mathrm{~s}$, are of type $\mathrm{T}$ and have a similar spin-down luminosity $(\log \dot{E})$ of 33.8 and 33.3 for B0919+06 and B2020+18, respectively.

\section{Spectral behavior of the ratio of the modes}

Because most data does not contain any information on the observed pulsar flux, the spectral index of the modes could only be determined relatively. Nonetheless, Figs. 22 through 24 show that in many cases there is a clear trend in the ratio of the average intensity of the two modes. The suggested power laws are listed in Table 1. These trends imply that the spectral indices of the two polarisation modes often differ from each other. Possible explanations are that the conversion of ordinary modes into extraordinary modes is frequency dependent (or in the case of radius to frequency mapping, height dependent), the two modes are damped differently, or one of the modes is moving out of the field of view.

\section{Association of modes with components}

Assuming that the different modes follow different paths in the magnetosphere, each mode can be responsible for several components in the total intensity profile. In many pulsars a mode can, at some frequencies, indeed be associated with one or more components of the average intensity (see Table 1). Figures 4 through 21 show that this can occur at any frequency. This association of polarisation modes with components suggests that the method used to separate the two polarisation modes is successful.

\section{Jump in the $P A$}

In several pulsars, such as $\mathrm{B} 0950+08$ (see Table 1), the PA jumps (sometimes gradual) $60^{\circ}$ through $90^{\circ}$, while at the same longitudes the ellipticity angle does not change sign and the intensity plots of the two modes do not allow for a smooth change in dominance, suggesting that there is no mode-change. Yet this behaviour does seem to be associated with modechanging, because the ellipticity angle usually shows a similar (but smaller) jump and the modes are of equal strength during such a jump. A partial (less than $90^{\circ}$ ) or gradual jump in the PA cannot be explained with two completely orthogonal modes, since the PA is completely determined by the strongest mode and can therefore only jump by $90^{\circ}$. In the view of the ordinary and extraordinary modes being $100 \%$ polarised and responsible for all the polarising characteristics of pulsar emission, the partial or gradual jump in the PA can be explained when the two polarisation modes are not always completely orthogonal
(Edwards 2004). An assumption that the modes are not 100\% polarised would not explain these jumps.

\section{Correlation with type}

It is interesting that only one of the observed features, mode drifting, is correlated with the pulsar's type. And even this one case involves only two pulsars. Also, the many pulsars we find containing one component, dominated by one mode at low frequency, and increasing in intensity with increasing frequency, are of all types. This suggests that the mechanism behind the frequency behavior of the polarisation of pulsars is similar in both the core as in the (multiple) conal components.

\section{Conclusions}

We have determined the average pulse profiles of two polarisation modes for 18 pulsars at multiple frequencies, assuming that the modes are completely orthogonal and $100 \%$ polarised. We find many cases where each polarisation mode can be wholly associated with one component in the average intensity and cases where a $90^{\circ}$ phase jump in the PA is associated with the two polarisation modes changing dominance. This suggests that the two polarisation modes are successfully determined in these cases. In other cases where there is a (gradual) jump in the PA of less than $90^{\circ}$ it would appear that one of the assumptions enabling the determination of the modes is not always valid. The spectra of the ratio of the integrated intensity of the polarisation modes show that in many cases there is a trend for one of the modes to become stronger with increasing frequency. This can be both the mode which we have defined as strongest, as well as the weakest mode. We also find that the average profiles of the modes often differ from each other at different frequencies. In particular, we find that when a component is dominated by one mode at low frequency it tends to increase in intensity with increasing frequency with respect to the rest of the profile. Furthermore, out of 18 pulsars there are 2 pulsars that clearly show one polarisation mode changing its longitudinal position with frequency. From our results we cannot determine whether the longitudinal transitions of one polarisation mode into the other at a fixed frequency are the result of the $\mathrm{X}$ - and O-modes beams being separated due to refraction (Barnard \& Arons 1986), or due to switching between significant and insignificant conversion of O-mode into $\mathrm{X}$-mode (Petrova 2001). Both explanations can also account for some of the complex changes that occur in the average profiles of the polarisation modes over frequency, since plasma waves with different frequency will traverse different paths through the pulsar magnetosphere. In the model of Petrova (2001) complex changes in the average profile of the polarisation modes over frequency implies that there are differences between the plasma number densities in polarisation limiting regions corresponding to emission at different frequencies. Following the technique as described in Petrova (2003) and assuming radius to frequency mapping, calculation of the plasma density distributions from polarisation profiles at different frequencies will then yield changes in the plasma density both as a function of longitude as well as altitude. 
Acknowledgements. We would like to thank an anonymous referee for his/her useful comments. This research has made extensive use of the database of published pulse profiles maintained by the European Pulsar Network, available at: http://www.mpifr-bonn.mpg.de/ div/pulsar/data/

\section{References}

Arons, J., \& Barnard, J. J. 1986, ApJ, 302, 120

Backer, D. C., \& Rankin, J. M. 1980, ApJS, 42, 143

Barnard, J. J., \& Arons, J. 1986, ApJ, 302, 138

Bartel, N., Morris, D., Sieber, W., \& Hankins, T. H. 1982, ApJ, 258, 776

Cordes, J. M., Rankin, J., \& Backer, D. C. 1978, ApJ, 223, 961

Edwards, R. T. 2004, A\&A, 426, 677

Gil, J. A., Snakowski, J. K., \& Stinebring, D. R. 1991, A\&A, 242, 119

Gil, J. A., Lyne, A. G., Rankin, J. M., Snakowski, J. K., \& Stinebring, D. R. 1992, A\&A, 255, 181

Gould, D. M., \& Lyne, A. G. 1998, MNRAS, 301, 235

Guojun, Q., Manchester, R. N., Lyne, A. G., \& Gould, D. M. 1995, MNRAS, 274, 572

Izvekova, V. A., Kuzmin, A. D., Lyne, A. G., Shitov, Y. P., \& Smith, F. G. 1993, MNRAS, 261, 865

Kuzmin, A. D., Izvekova, V. A., Shitov, Y. P., et al. 1998, A\&AS, 127, 355
Manchester, R. N., Taylor, J. H., \& Huguenin, G. R. 1975, ApJ, 196, 83

McKinnon, M. M. 1997, ApJ, 475, 763

McKinnon, M. M., \& Stinebring, D. R. 1998, ApJ, 502, 883

McKinnon, M. M., \& Stinebring, D. R. 2000, ApJ, 529, 435

Petrova, S. A. 2001, A\&A, 378, 883

Petrova, S. A. 2003, A\&A, 408, 1057

Radhakrishnan, V., \& Cooke, D. J. 1969, Astrophys. Lett., 3, 225

Rankin, J. M. 1983, ApJ, 274, 333

Rankin, J. M. 1993, ApJ, 405, 285

Rankin, J. M., Wolszczan, A., \& Stinebring, D. R. 1988, ApJ, 324, 1048

Rankin, J. M., Ramachandran, R., \& Suleymanova, S. A. 2004 [arXiv: astro-ph/0406239]

Seiradakis, J. H., Gil, J. A., Graham, D. A., et al. 1995, A\&AS, 111, 205

Sieber, W., Reinecke, R., \& Wielebinski, R. 1975, A\&A, 38, 169

Stinebring, D. R., Cordes, J. M., Rankin, J. M., Weisberg, J. M., \& Boriakoff, V. 1984, ApJS, 55, 247

Taylor, J. H., Manchester, R. N., \& Lyne, A. G. 1993, ApJS, 88, 529

von Hoensbroech, A., Kijak, J., \& Krawczyk, A. 1998, A\&A, 334, 571

von Hoensbroech, A., \& Xilouris, K. M. 1997, A\&AS, 126, 121

Wu, X., Manchester, R. N., Lyne, A. G., \& Qiao, G. 1993, MNRAS, 261,630 
J. M. Smits et al.: Frequency dependence of orthogonal polarisation modes in pulsars, Online Material p 1

\section{Online Material}


J. M. Smits et al.: Frequency dependence of orthogonal polarisation modes in pulsars, Online Material $p 2$
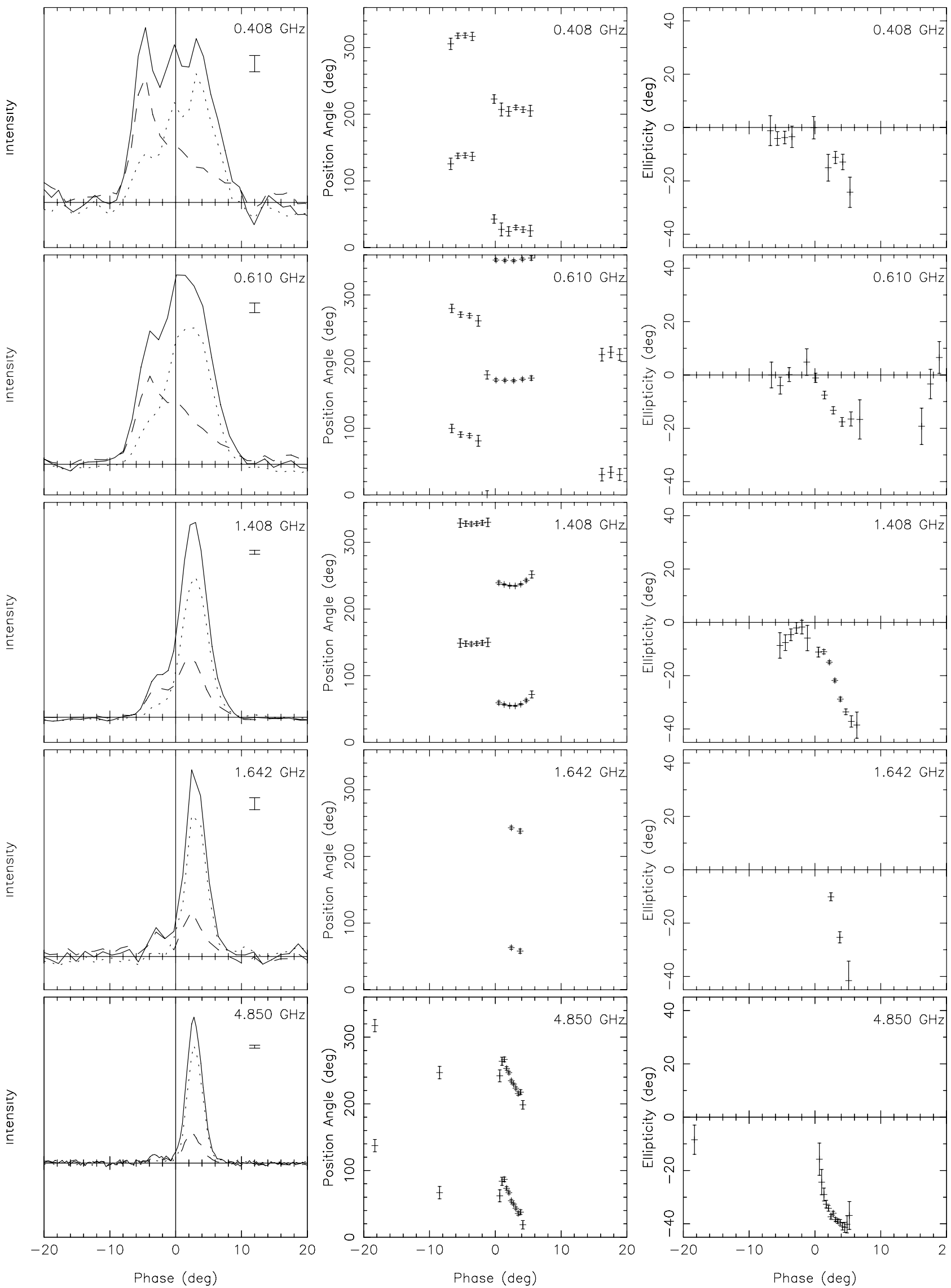

Fig. 4. Total Intensity, intensity of orthogonal modes, position angle and ellipticity angle as a function of frequency of PSR B0144+59. The error bars represent the noiselevel, given by the rms of the off-pulse region of the profile. 
J. M. Smits et al.: Frequency dependence of orthogonal polarisation modes in pulsars, Online Material p 3
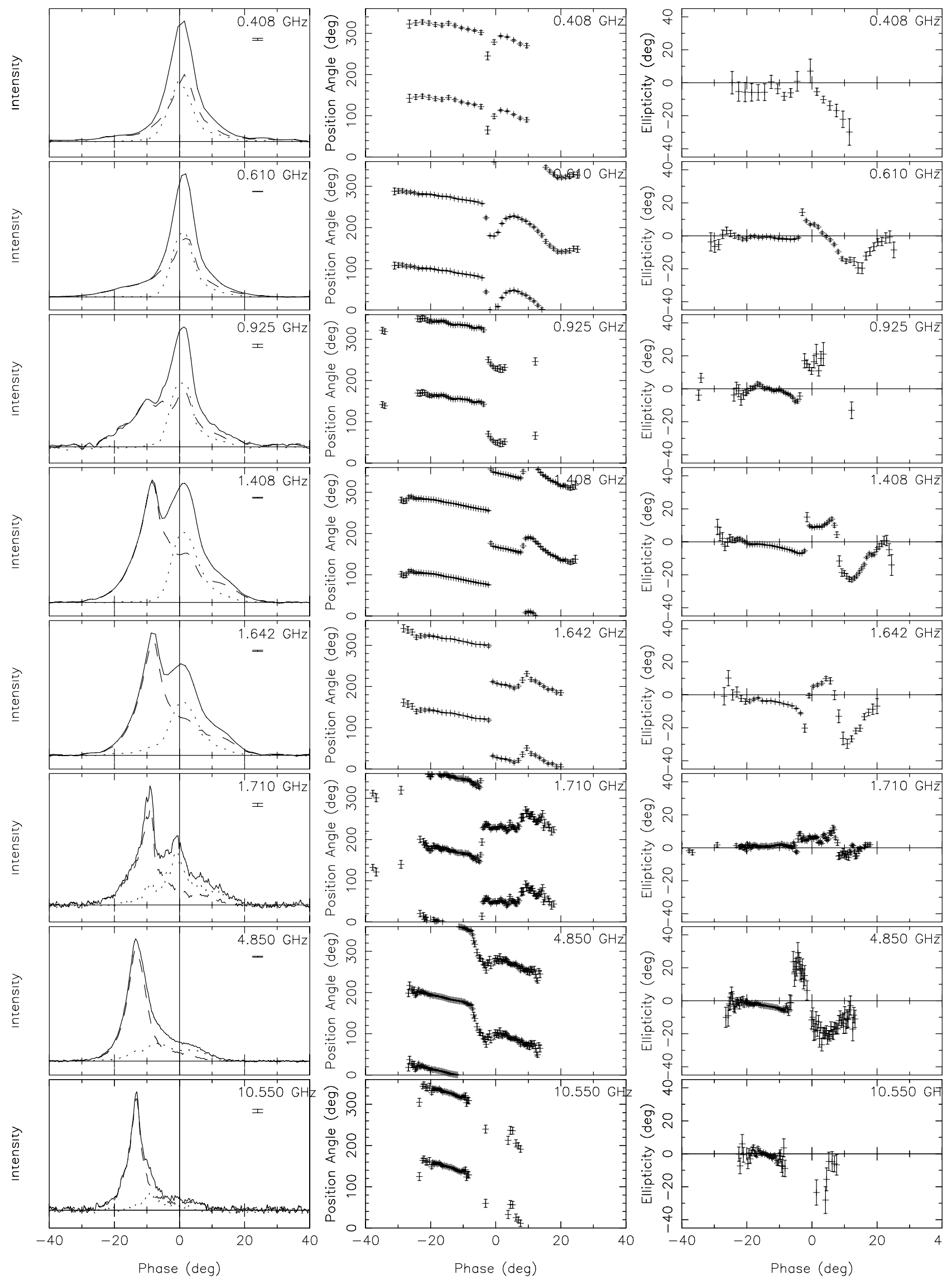

Fig. 5. Total Intensity, intensity of orthogonal modes, position angle and ellipticity angle as a function of frequency of PSR B0355+54. The error bars represent the noiselevel, given by the rms of the off-pulse region of the profile. 
J. M. Smits et al.: Frequency dependence of orthogonal polarisation modes in pulsars, Online Material p 4
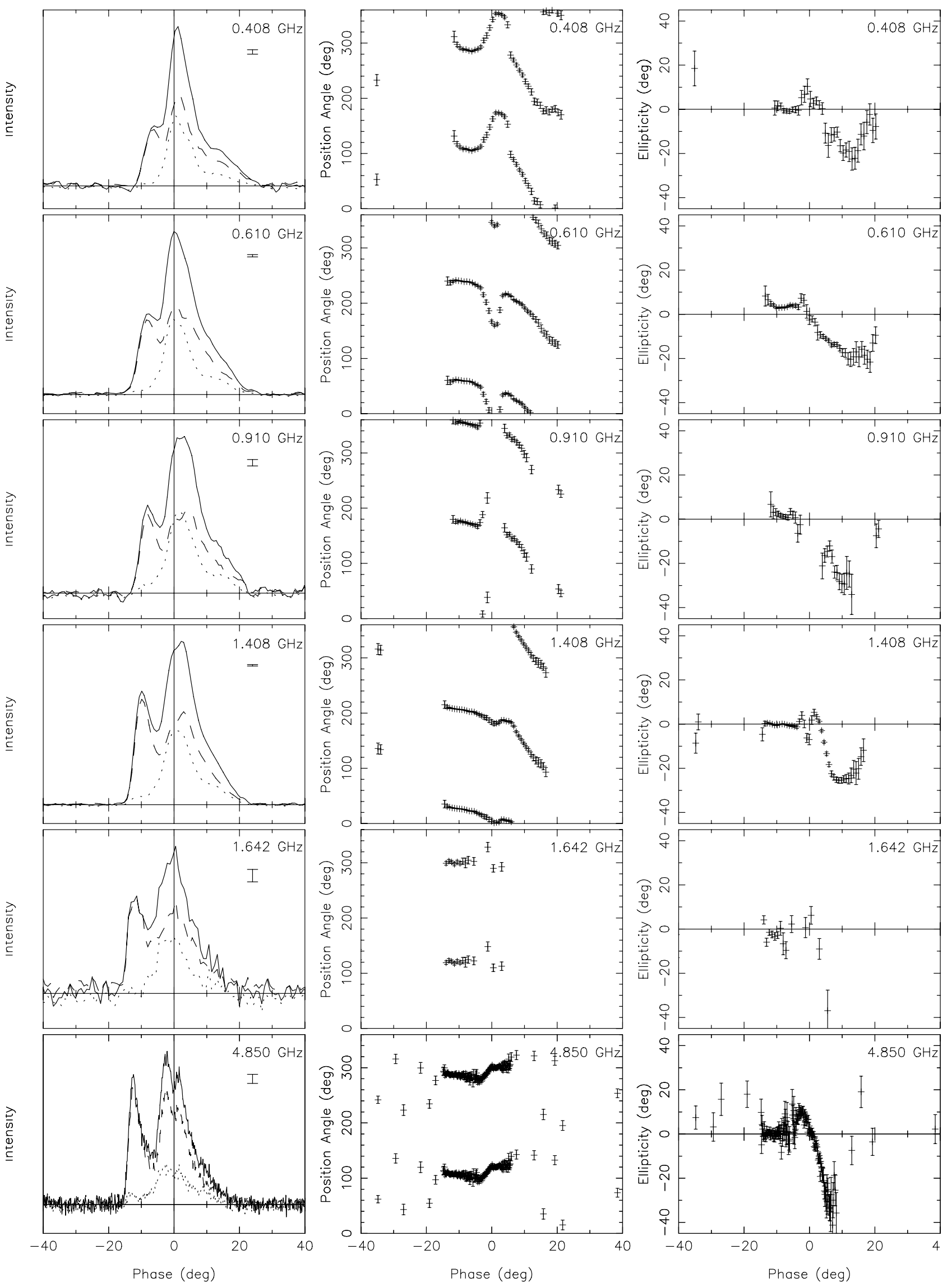

Fig. 6. Total Intensity, intensity of orthogonal modes, position angle and ellipticity angle as a function of frequency of PSR B0450+55. The error bars represent the noiselevel, given by the rms of the off-pulse region of the profile. 
J. M. Smits et al.: Frequency dependence of orthogonal polarisation modes in pulsars, Online Material p 5
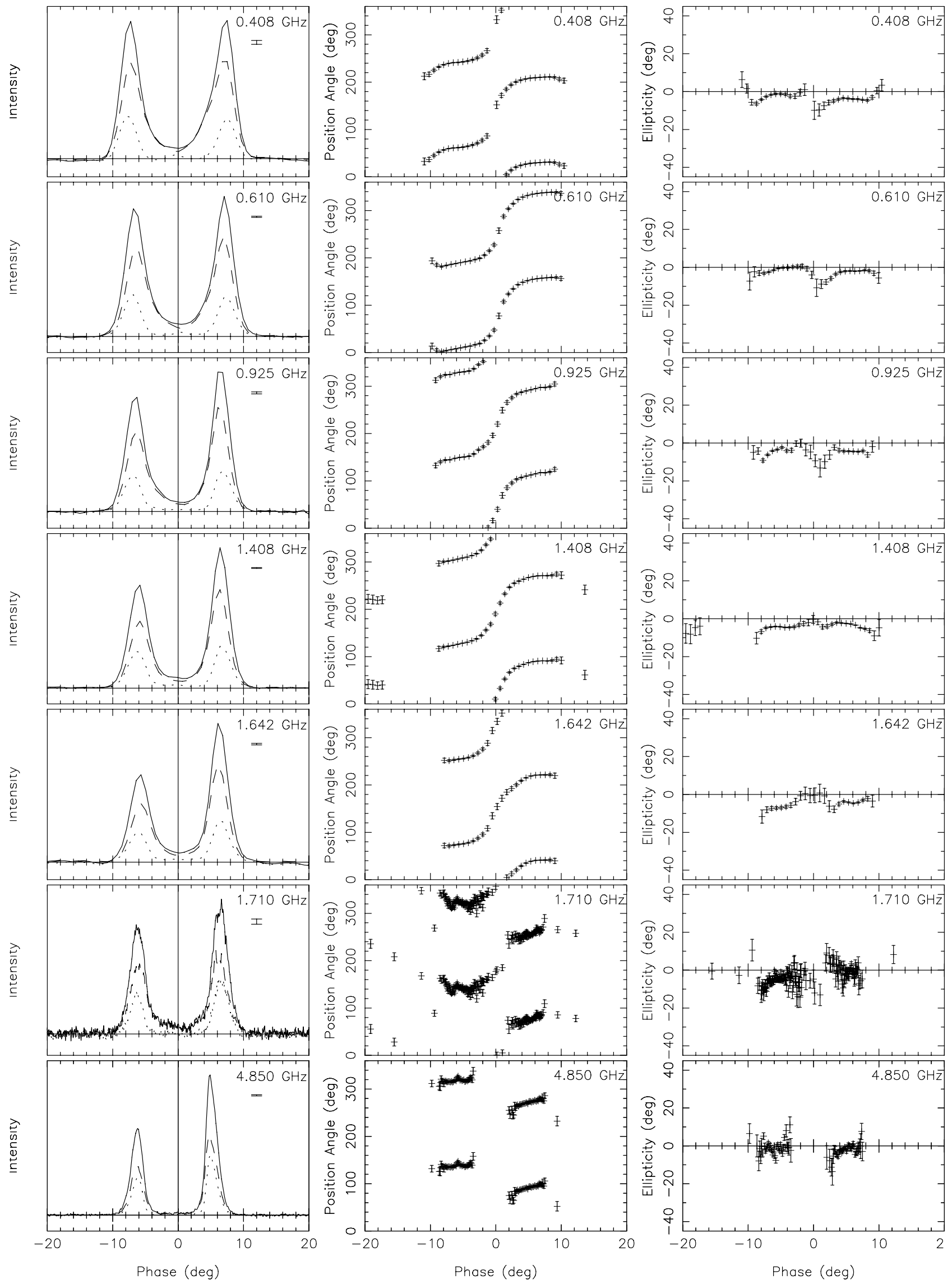

Fig. 7. Total Intensity, intensity of orthogonal modes, position angle and ellipticity angle as a function of frequency of PSR B0525+21. The error bars represent the noiselevel, given by the rms of the off-pulse region of the profile. 
J. M. Smits et al.: Frequency dependence of orthogonal polarisation modes in pulsars, Online Material p 6
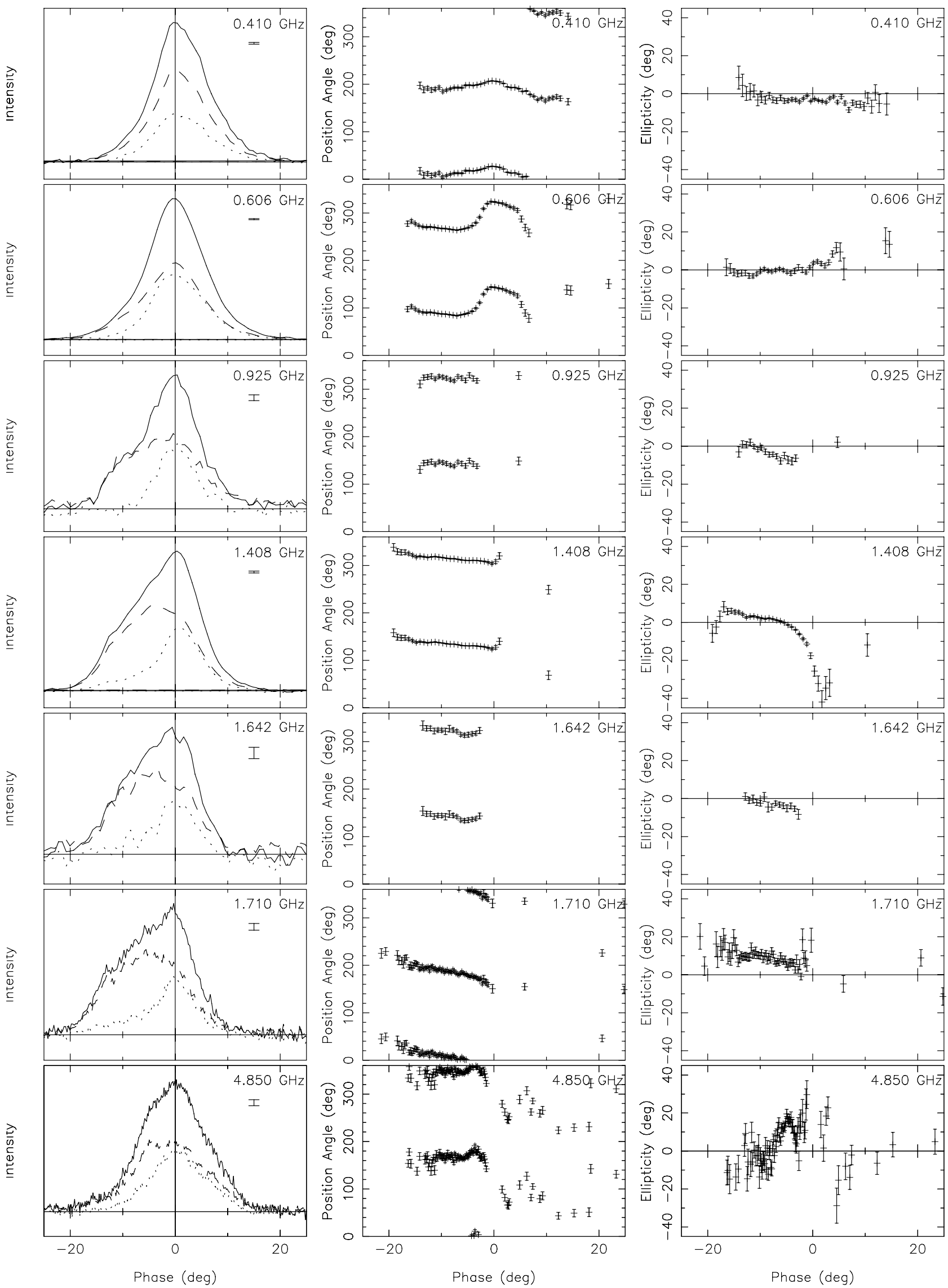

Fig. 8. Total Intensity, intensity of orthogonal modes, position angle and ellipticity angle as a function of frequency of PSR B0809+74. The error bars represent the noiselevel, given by the rms of the off-pulse region of the profile. 
J. M. Smits et al.: Frequency dependence of orthogonal polarisation modes in pulsars, Online Material $p 7$
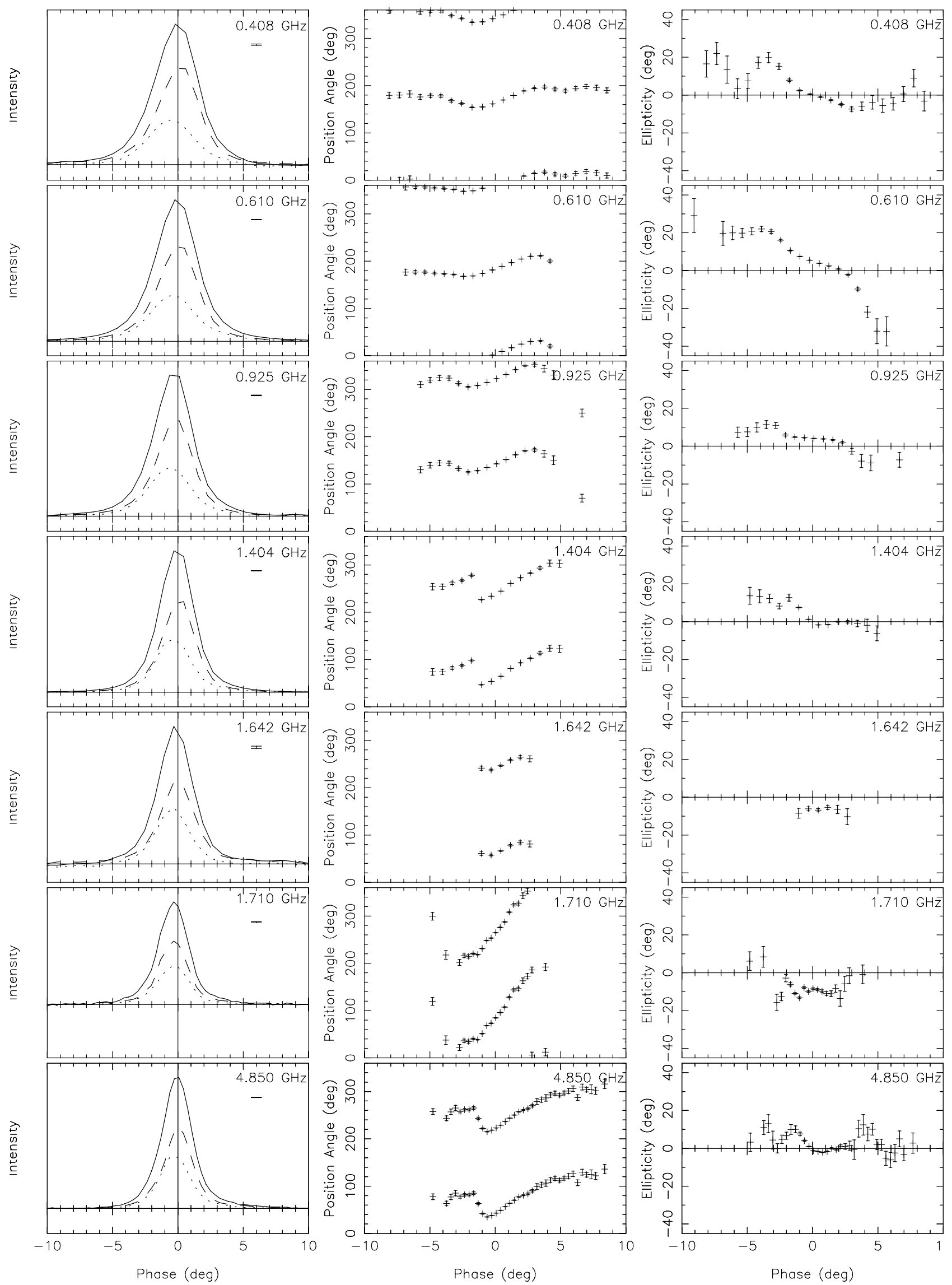

Fig. 9. Total Intensity, intensity of orthogonal modes, position angle and ellipticity angle as a function of frequency of PSR B0823+26. The error bars represent the noiselevel, given by the rms of the off-pulse region of the profile. 
J. M. Smits et al.: Frequency dependence of orthogonal polarisation modes in pulsars, Online Material p 8
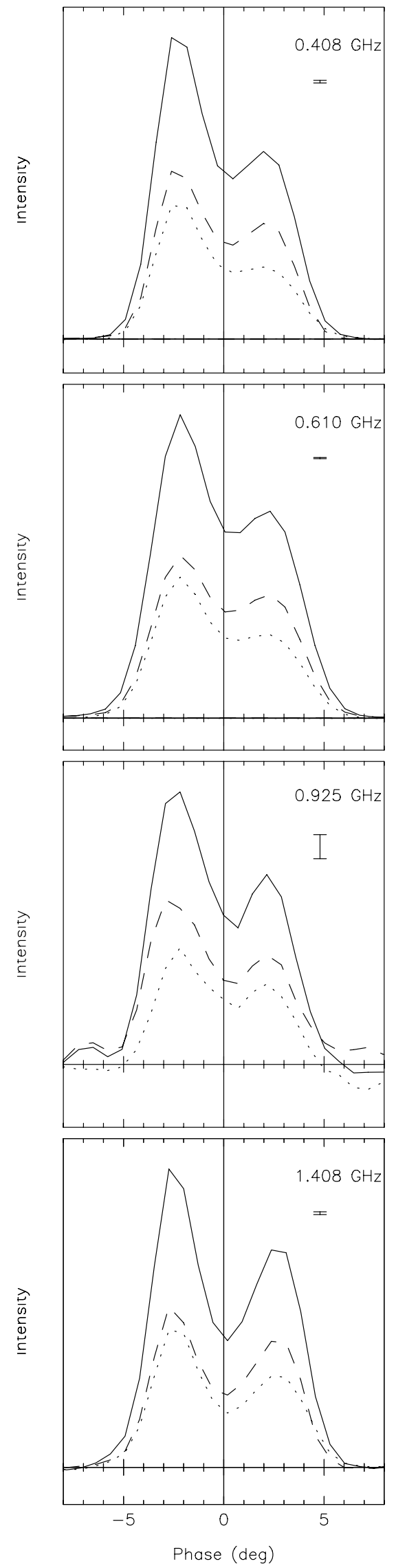
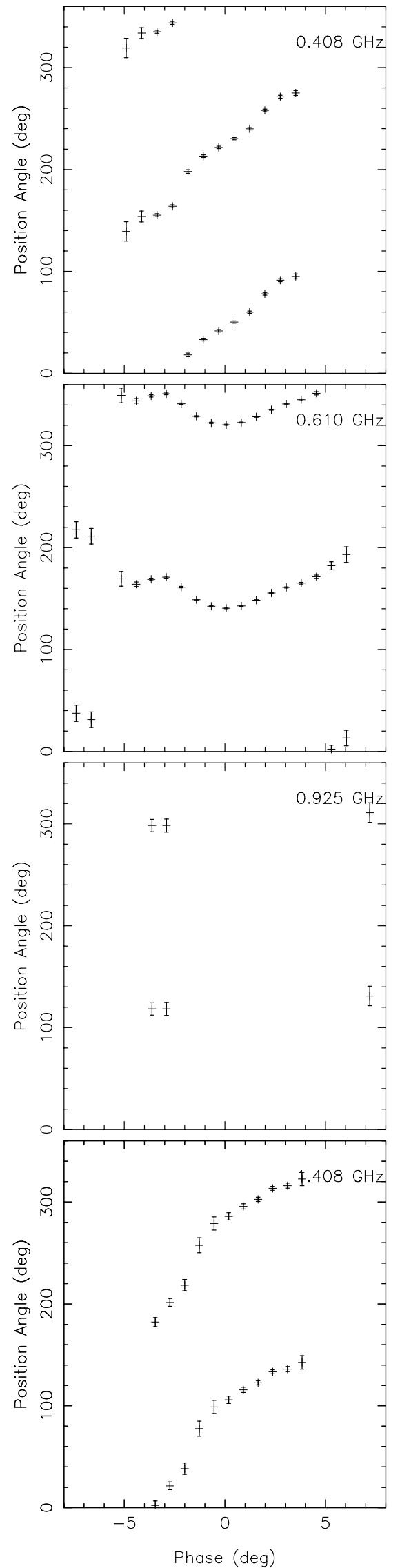
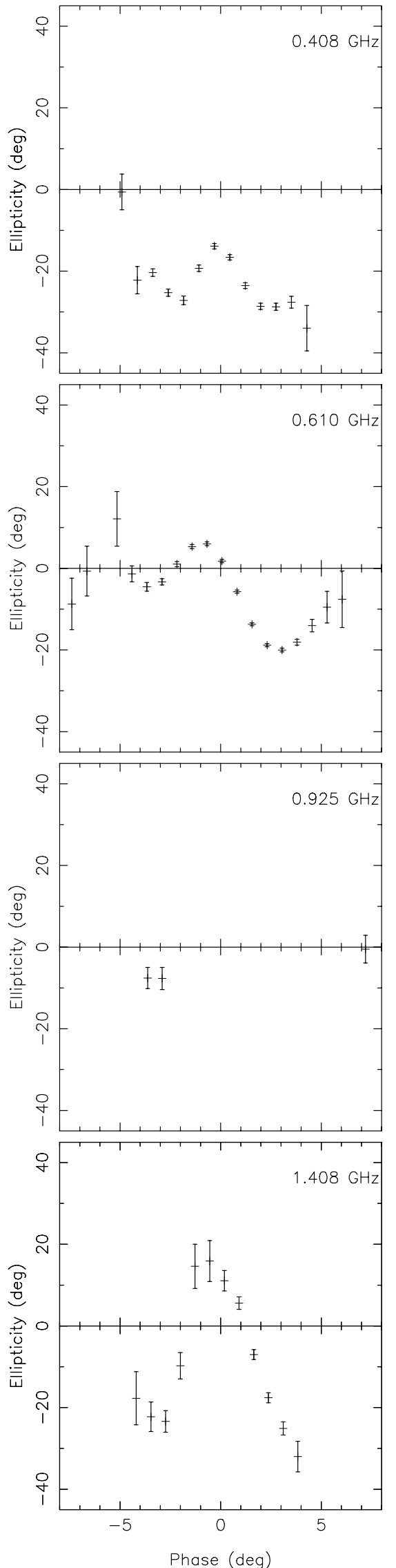

Fig. 10. Total Intensity, intensity of orthogonal modes, position angle and ellipticity angle as a function of frequency of PSR B0834+06. The error bars represent the noiselevel, given by the rms of the off-pulse region of the profile. 
J. M. Smits et al.: Frequency dependence of orthogonal polarisation modes in pulsars, Online Material $p 9$
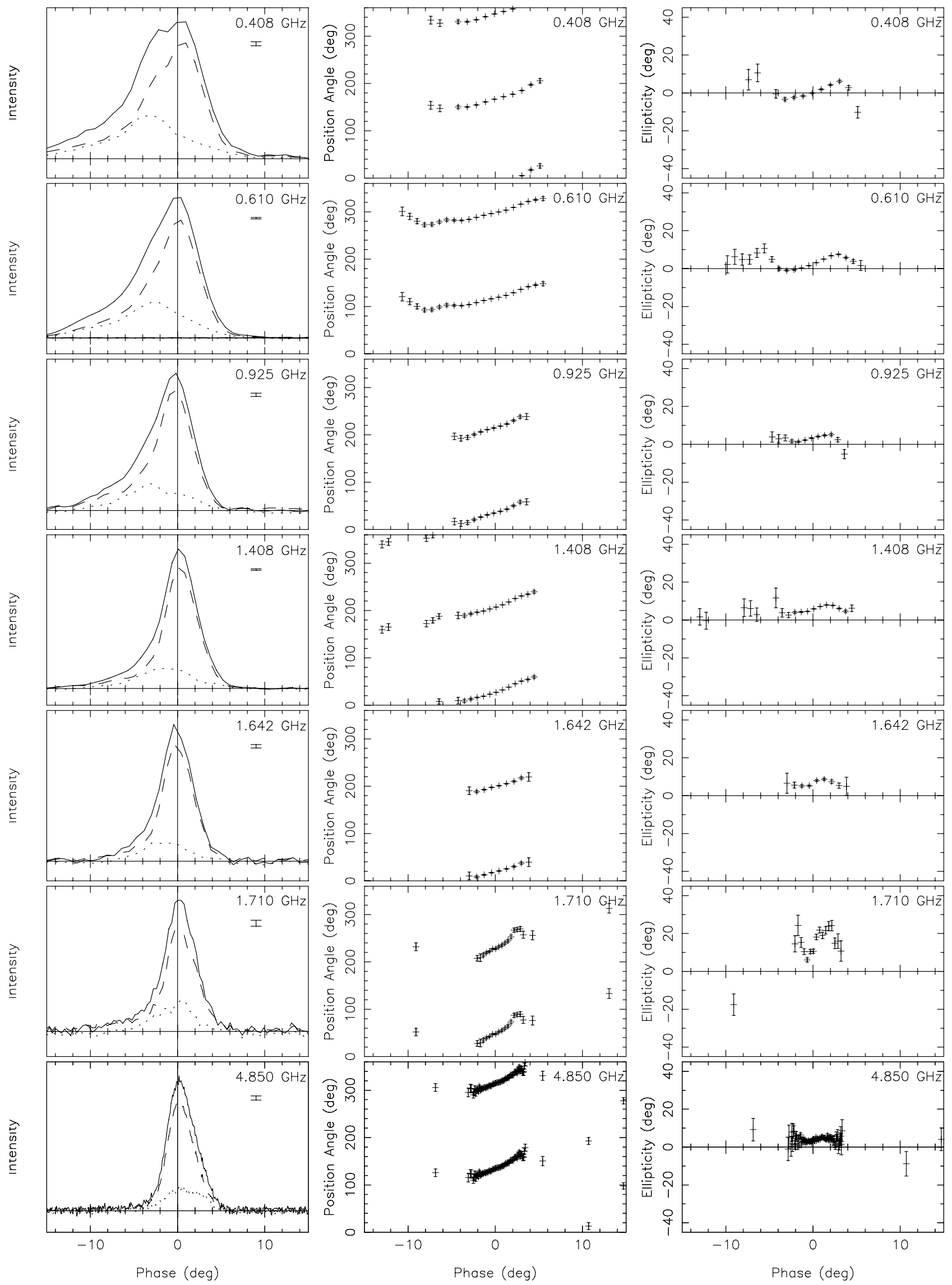

Fig. 11. Total Intensity, intensity of orthogonal modes, position angle and ellipticity angle as a function of frequency of PSR B0919+06. The error bars represent the noiselevel, given by the rms of the off-pulse region of the profile. 
J. M. Smits et al.: Frequency dependence of orthogonal polarisation modes in pulsars, Online Material p 10
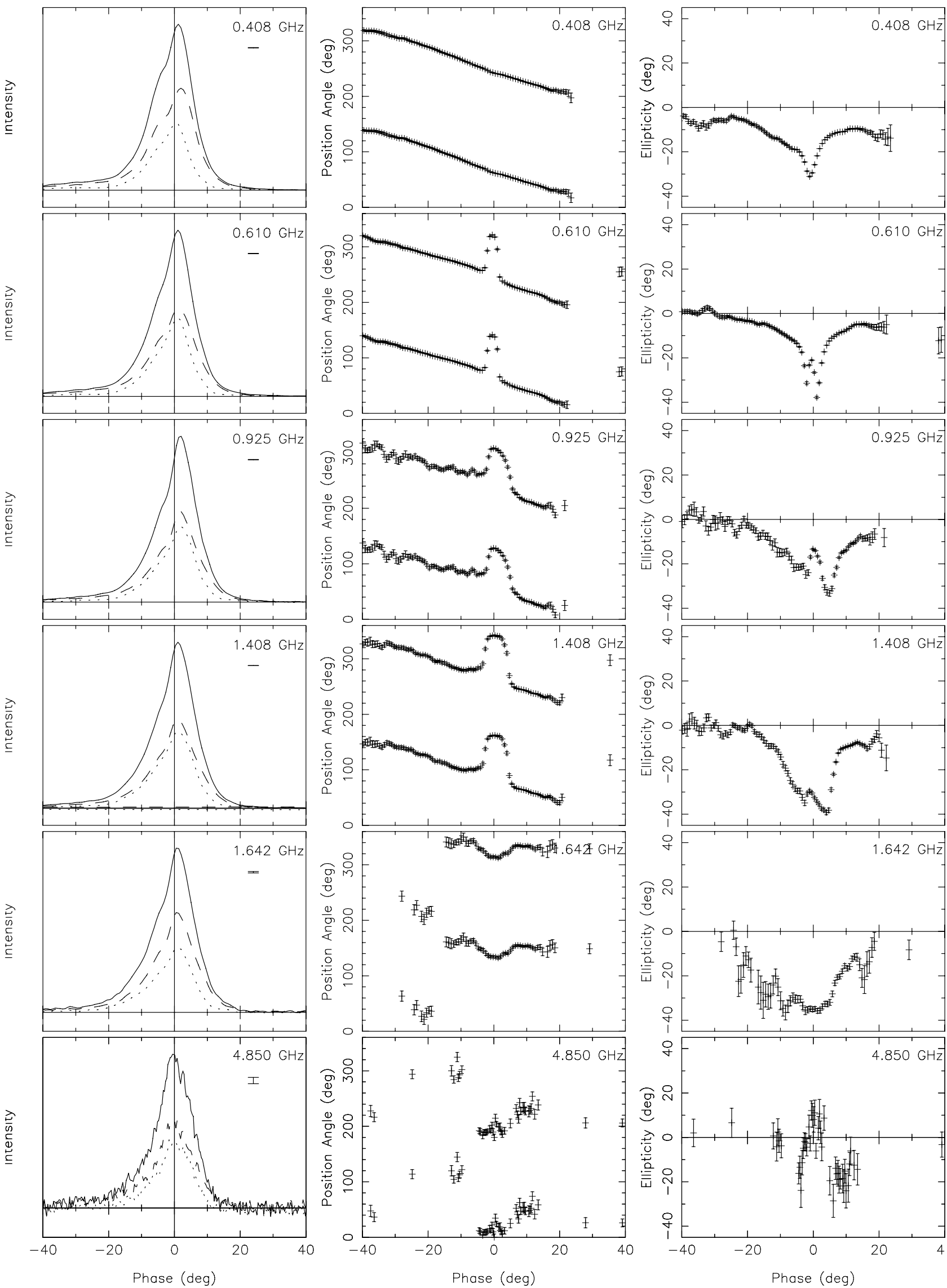

Fig. 12. Total Intensity, intensity of orthogonal modes, position angle and ellipticity angle as a function of frequency of PSR B0950+08. The error bars represent the noiselevel, given by the rms of the off-pulse region of the profile. 
J. M. Smits et al.: Frequency dependence of orthogonal polarisation modes in pulsars, Online Material p 11
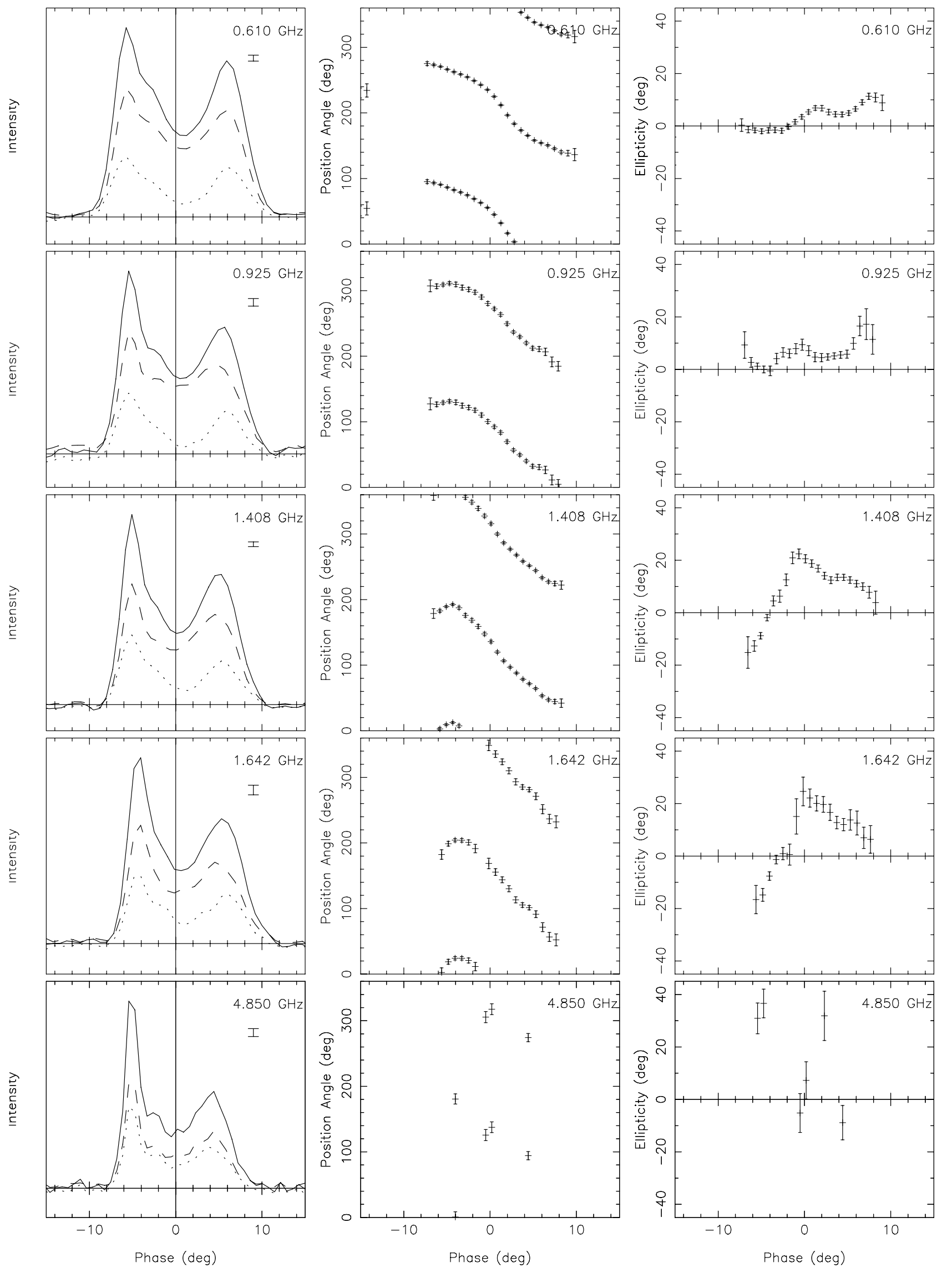

Fig. 13. Total Intensity, intensity of orthogonal modes, position angle and ellipticity angle as a function of frequency of PSR B1039-19. The error bars represent the noiselevel, given by the rms of the off-pulse region of the profile. 
J. M. Smits et al.: Frequency dependence of orthogonal polarisation modes in pulsars, Online Material p 12
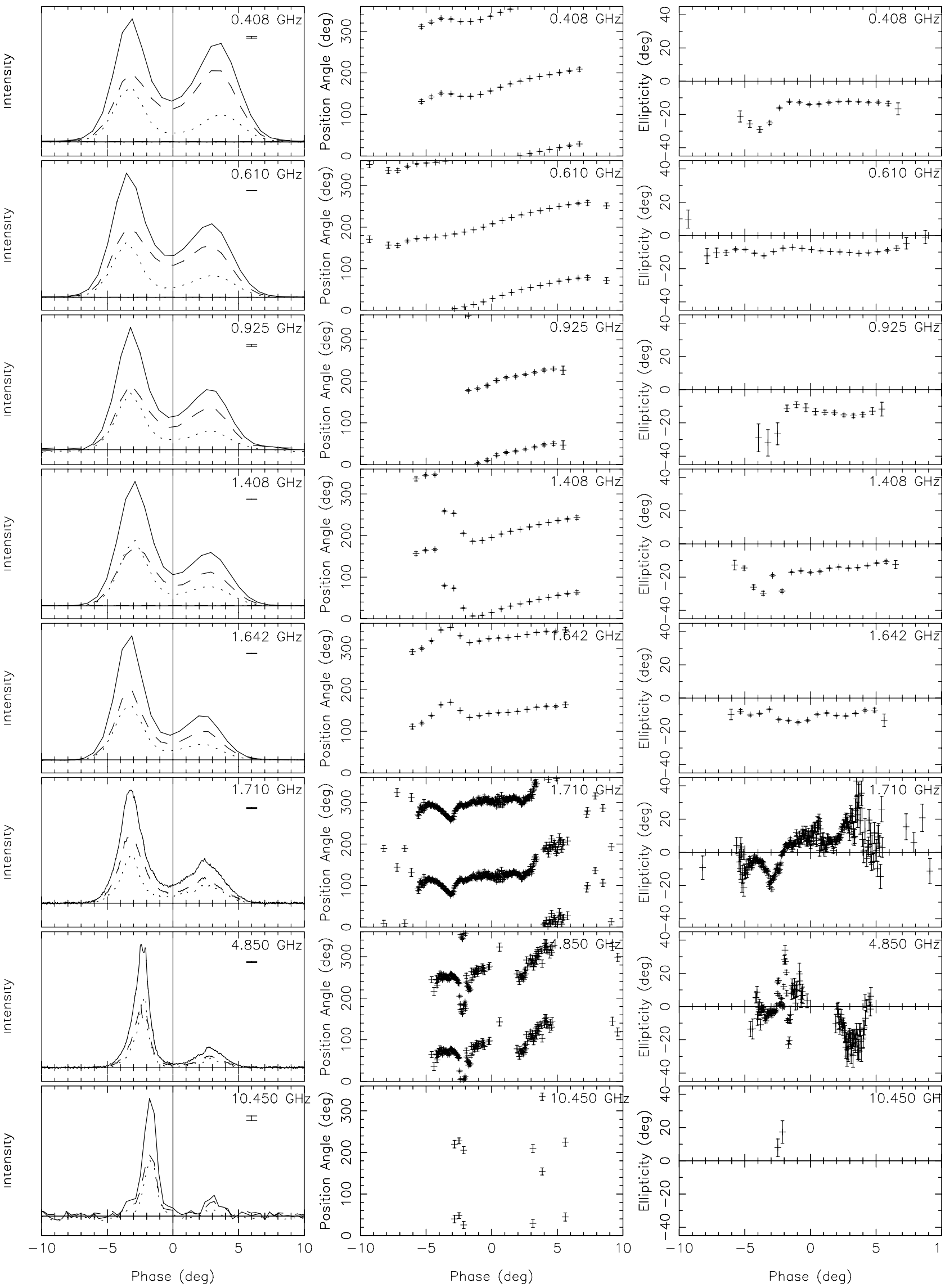

Fig. 14. Total Intensity, intensity of orthogonal modes, position angle and ellipticity angle as a function of frequency of PSR B1133+16. The error bars represent the noiselevel, given by the rms of the off-pulse region of the profile. 
J. M. Smits et al.: Frequency dependence of orthogonal polarisation modes in pulsars, Online Material p 13
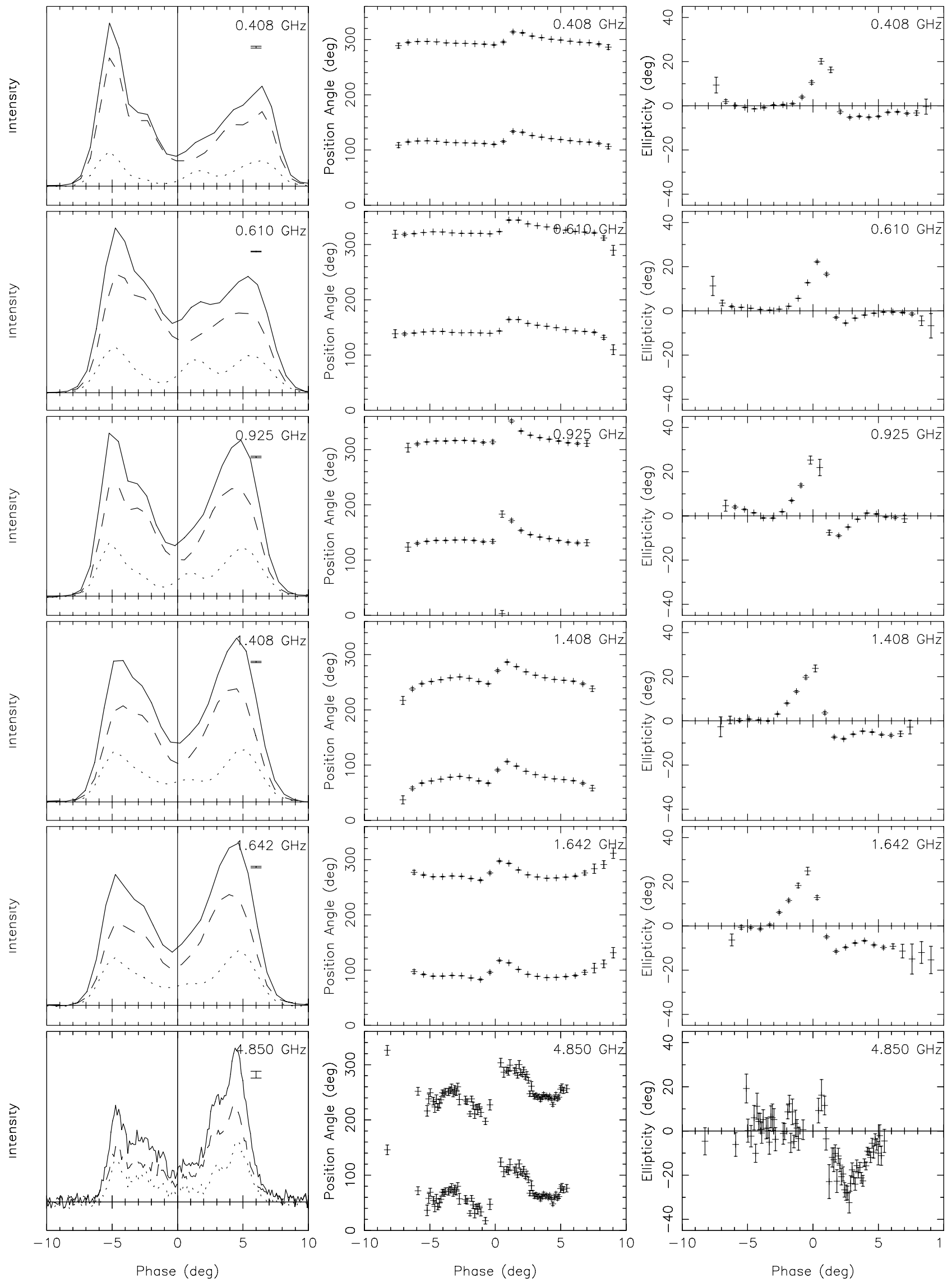

Fig. 15. Total Intensity, intensity of orthogonal modes, position angle and ellipticity angle as a function of frequency of PSR B1237+25. The error bars represent the noiselevel, given by the rms of the off-pulse region of the profile. 
J. M. Smits et al.: Frequency dependence of orthogonal polarisation modes in pulsars, Online Material p 14
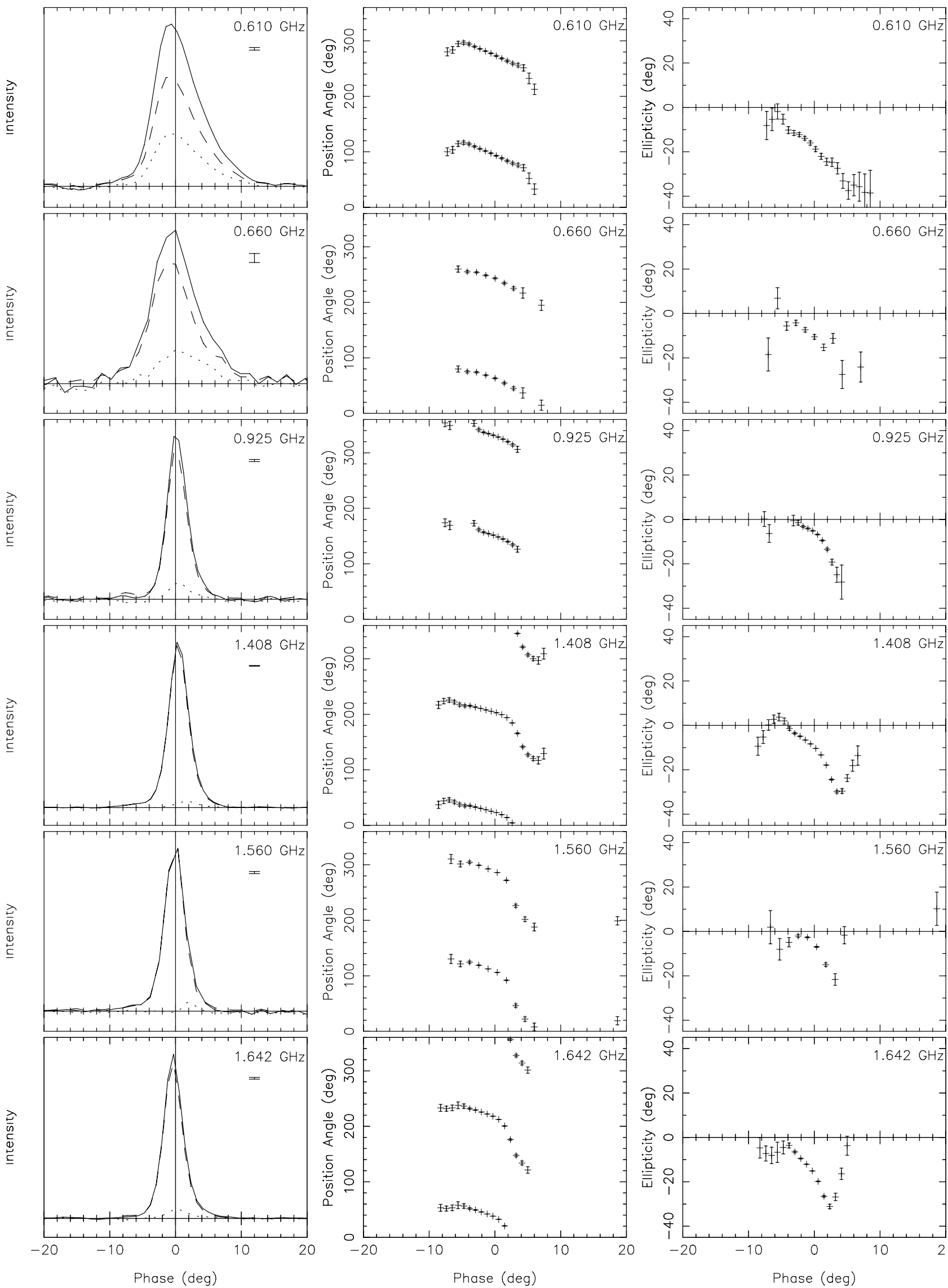

Fig. 16. Total Intensity, intensity of orthogonal modes, position angle and ellipticity angle as a function of frequency of PSR B1737-30. The error bars represent the noiselevel, given by the rms of the off-pulse region of the profile. 
J. M. Smits et al.: Frequency dependence of orthogonal polarisation modes in pulsars, Online Material p 15
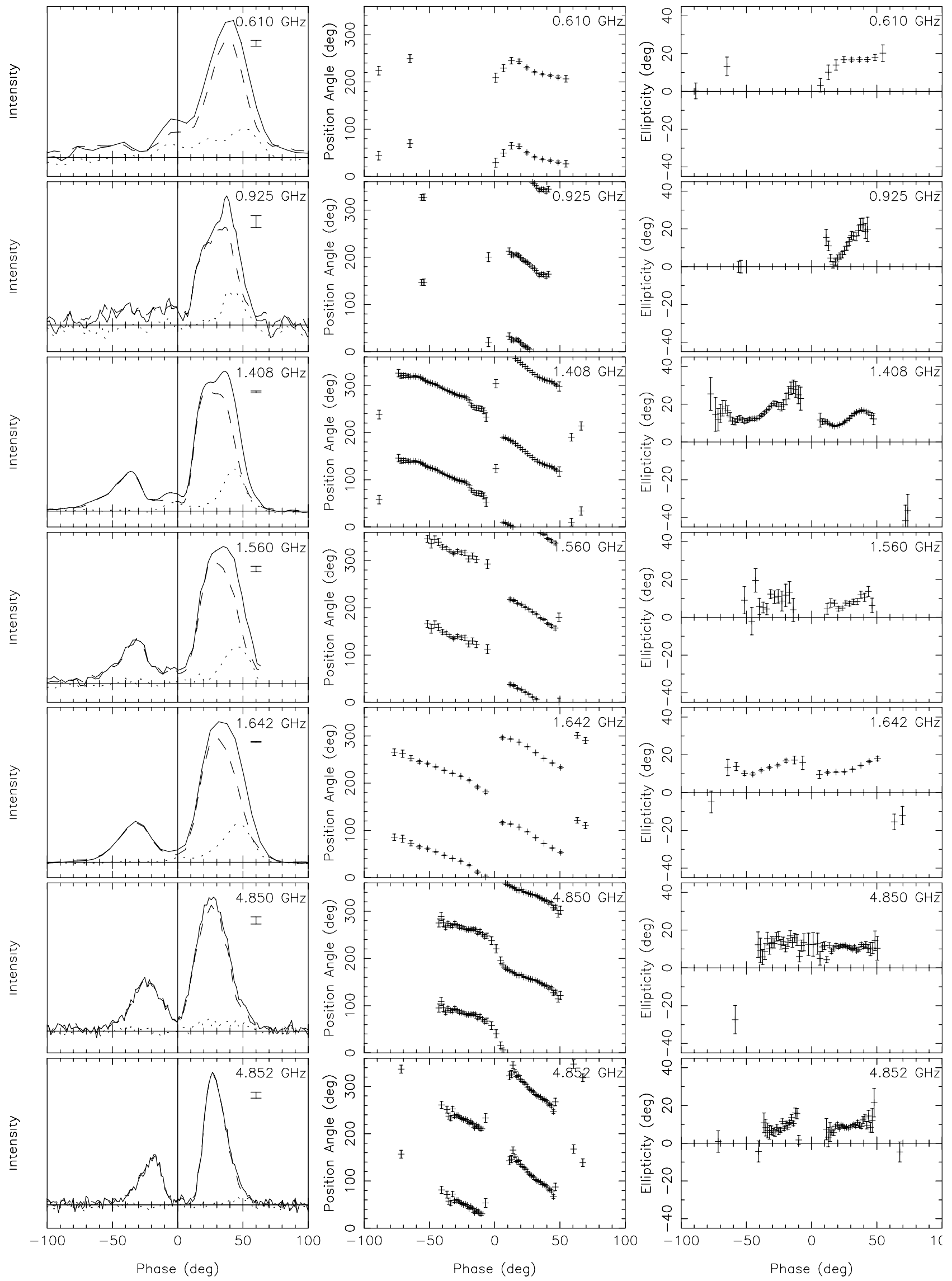

Fig. 17. Total Intensity, intensity of orthogonal modes, position angle and ellipticity angle as a function of frequency of PSR B1800-21. The error bars represent the noiselevel, given by the rms of the off-pulse region of the profile. 
J. M. Smits et al.: Frequency dependence of orthogonal polarisation modes in pulsars, Online Material p 16
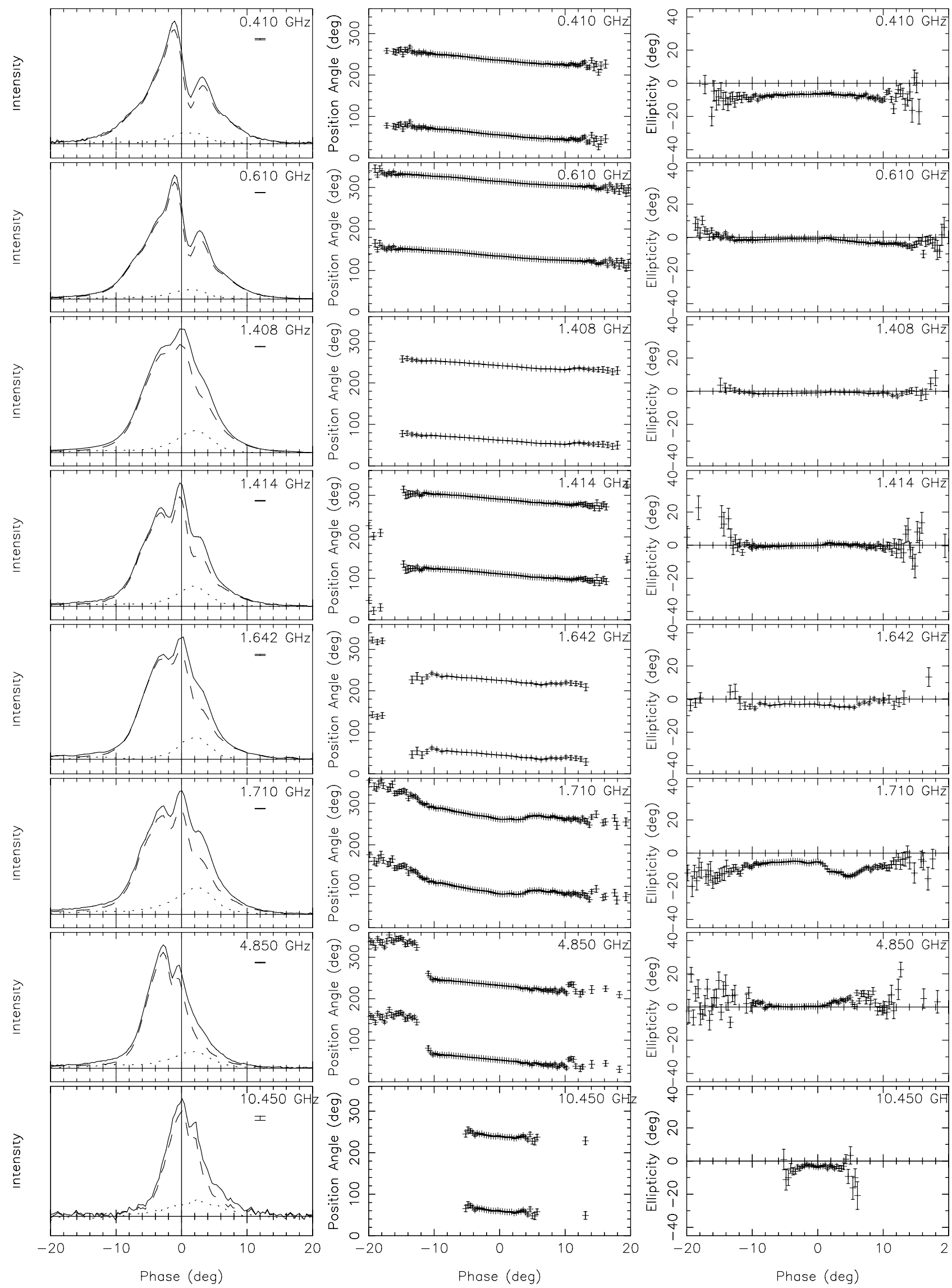

Fig. 18. Total Intensity, intensity of orthogonal modes, position angle and ellipticity angle as a function of frequency of PSR B1929+10. The error bars represent the noiselevel, given by the rms of the off-pulse region of the profile. 
J. M. Smits et al.: Frequency dependence of orthogonal polarisation modes in pulsars, Online Material p 17
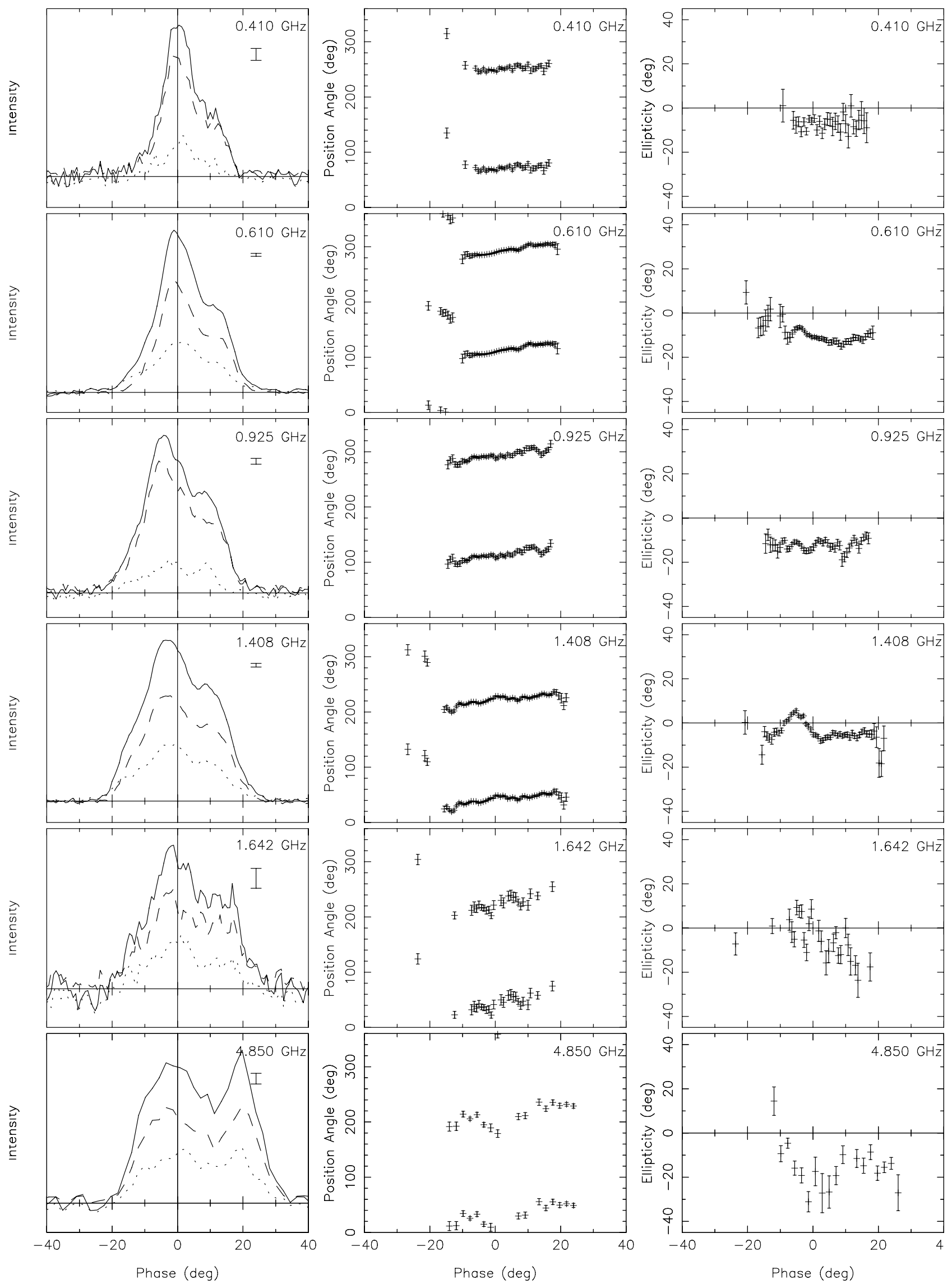

Fig. 19. Total Intensity, intensity of orthogonal modes, position angle and ellipticity angle as a function of frequency of PSR B1944+17. The error bars represent the noiselevel, given by the rms of the off-pulse region of the profile. 
J. M. Smits et al.: Frequency dependence of orthogonal polarisation modes in pulsars, Online Material p 18
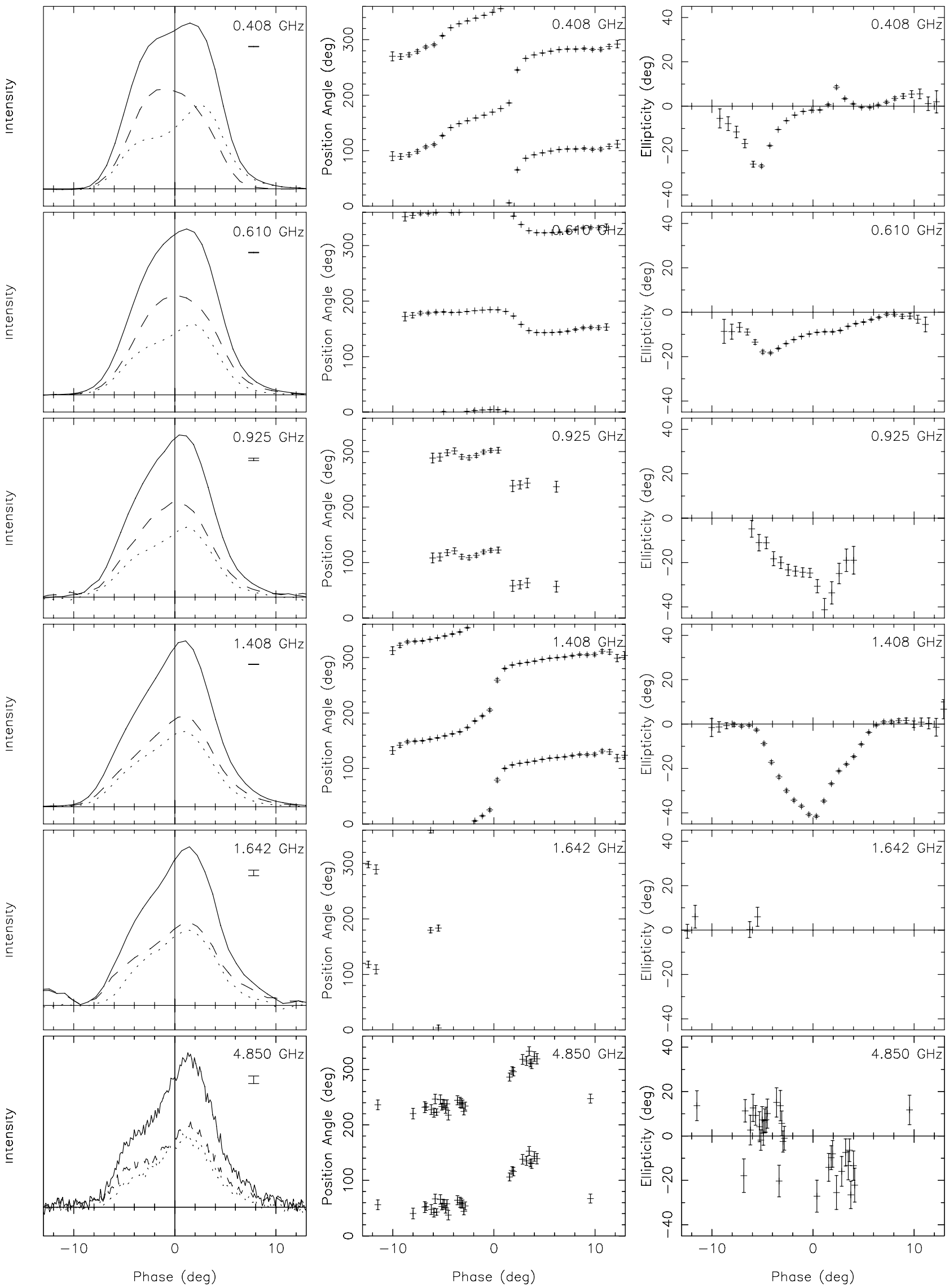

Fig. 20. Total Intensity, intensity of orthogonal modes, position angle and ellipticity angle as a function of frequency of PSR B2016+28. The error bars represent the noiselevel, given by the rms of the off-pulse region of the profile. 
J. M. Smits et al.: Frequency dependence of orthogonal polarisation modes in pulsars, Online Material p 19
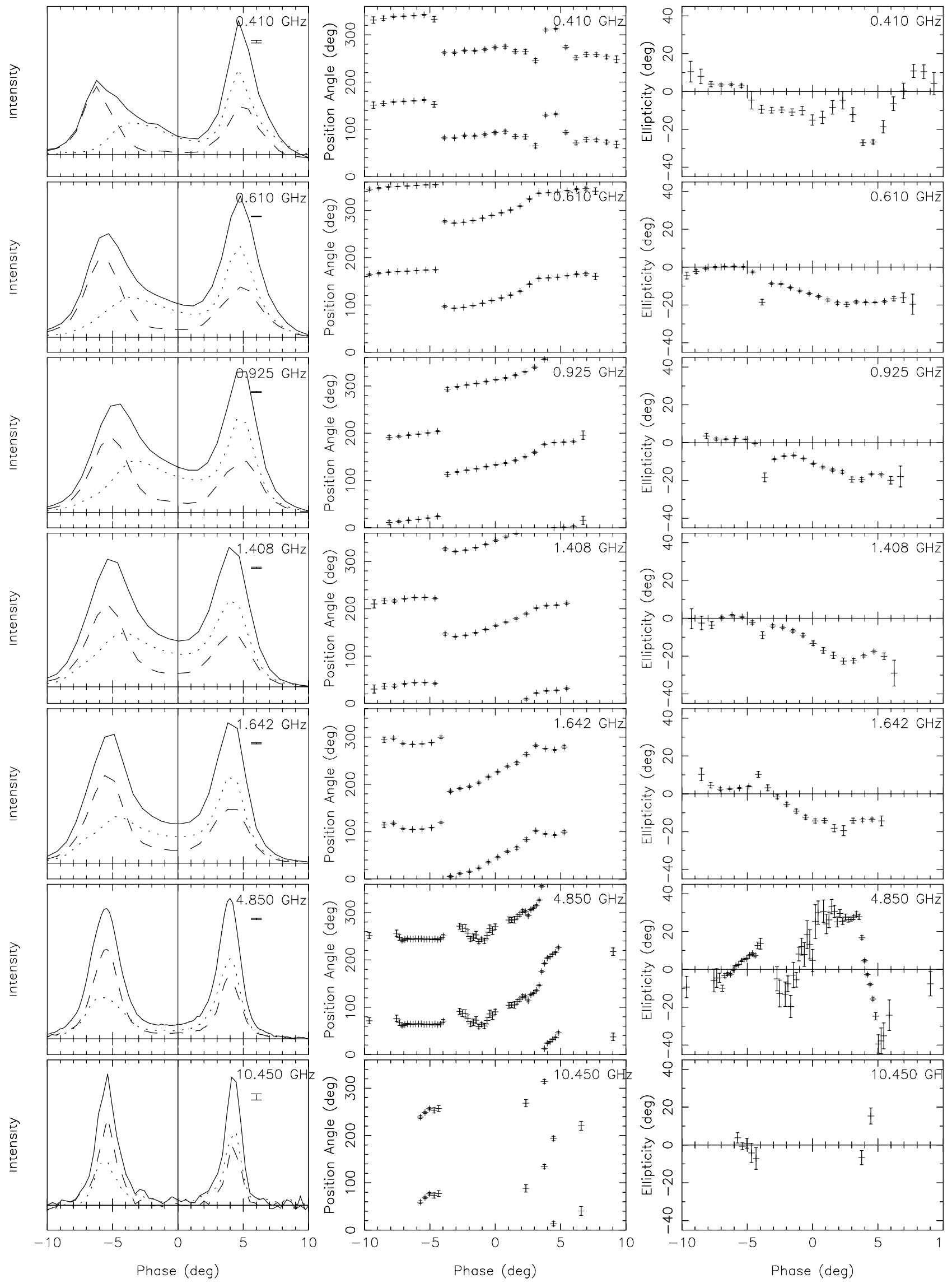

Fig. 21. Total Intensity, intensity of orthogonal modes, position angle and ellipticity angle as a function of frequency of PSR B2020+28. The error bars represent the noiselevel, given by the rms of the off-pulse region of the profile. 
J. M. Smits et al.: Frequency dependence of orthogonal polarisation modes in pulsars, Online Material p 20

B0144+59

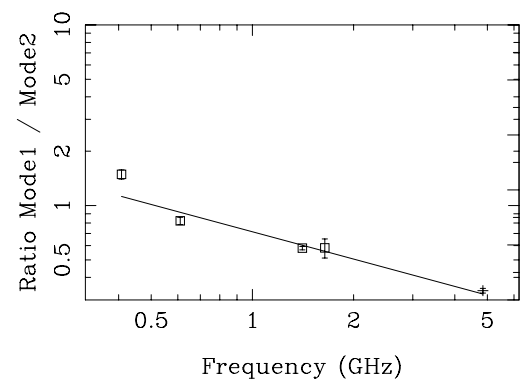

B0450+55 (right component)

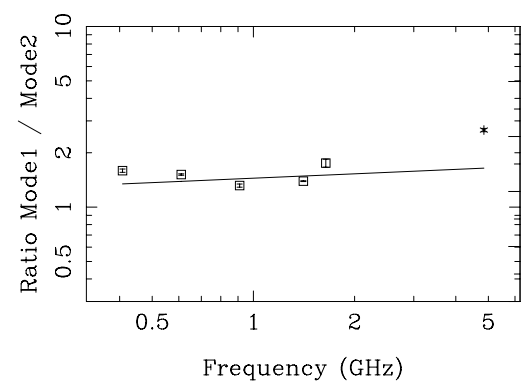

B0525+21 (right component)

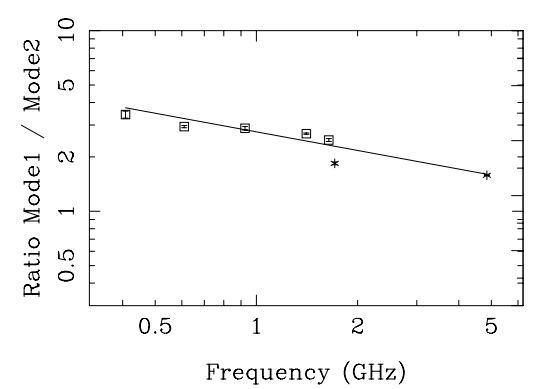

B0834+06 (both components)

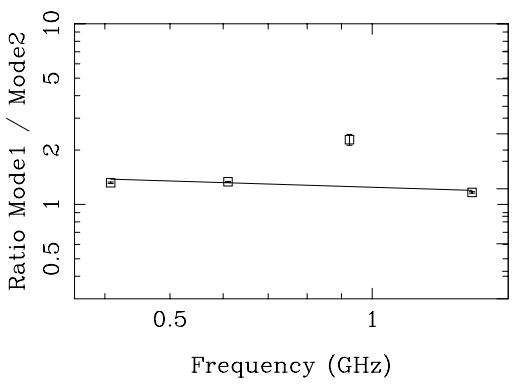

B0355+54

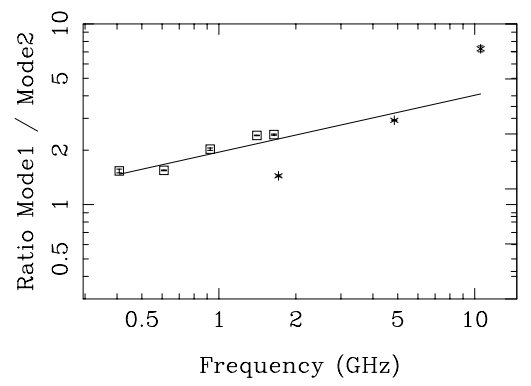

B0525+21 (both components)

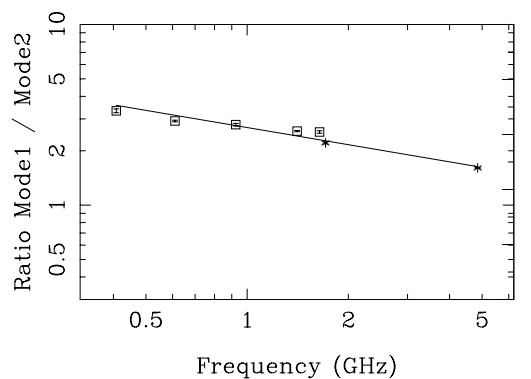

B0809+74

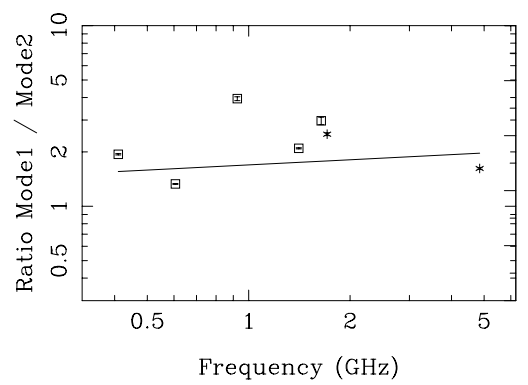

B0834+06 (left component)

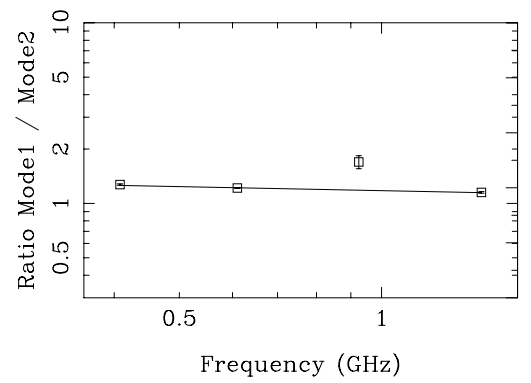

B0450+55 (both components)

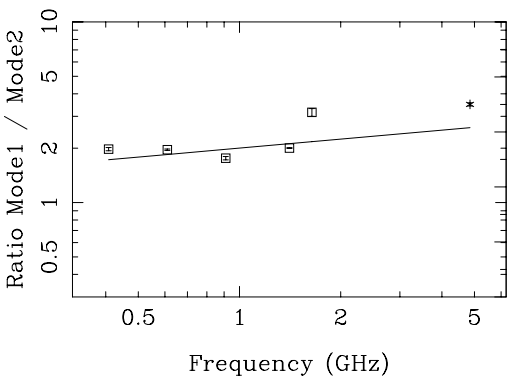

B0525+21 (left component)

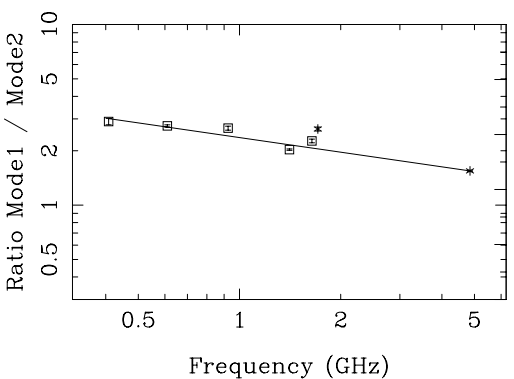

$\mathrm{B} 0823+26$

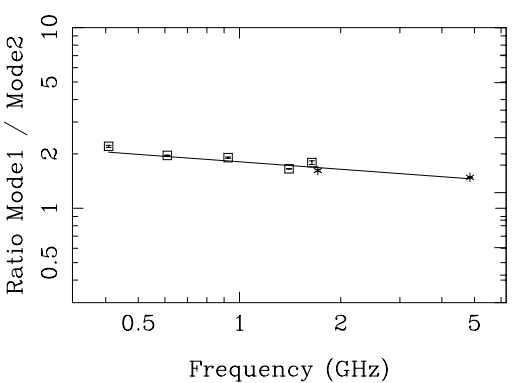

B0834+06 (right component)

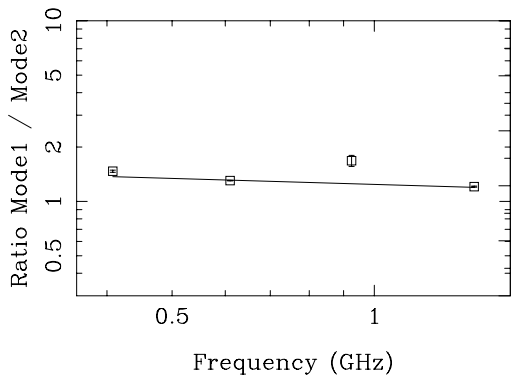

Fig. 22. The ratio of the average intensity of two orthogonally polarised modes as a function of frequency, plotted on a log-log scale, for all pulsars. The error is calculated as $\sigma_{I}=\sqrt{N} \cdot \sigma$, where $N$ is the number of bins of the profile and $\sigma$ is the noise level of the average intensity profile. The line through the points is the best straight line fit. Note that the scales on the vertical axis are chosen to be the same as to allow for comparison of the slopes. 
J. M. Smits et al.: Frequency dependence of orthogonal polarisation modes in pulsars, Online Material p 21

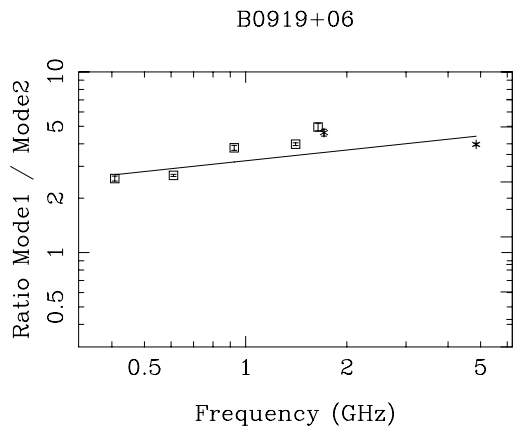

B1039-19 (left component)

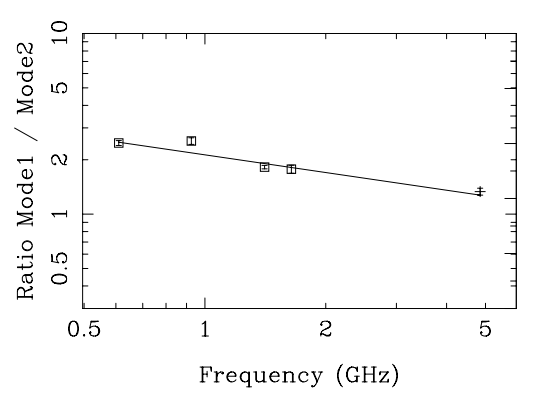

B1133+16 (left component)

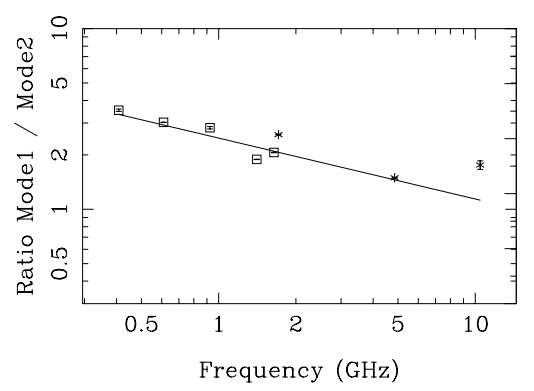

B1237+25 (left component)

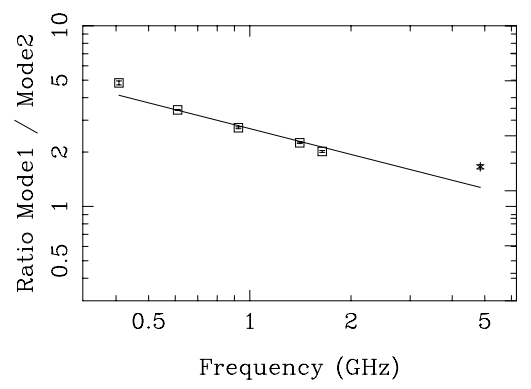

B0950+08

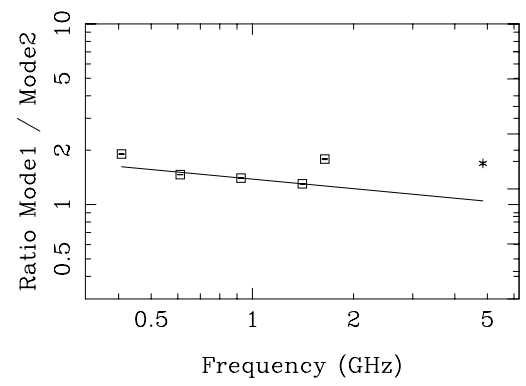

B1039-19 (right component)

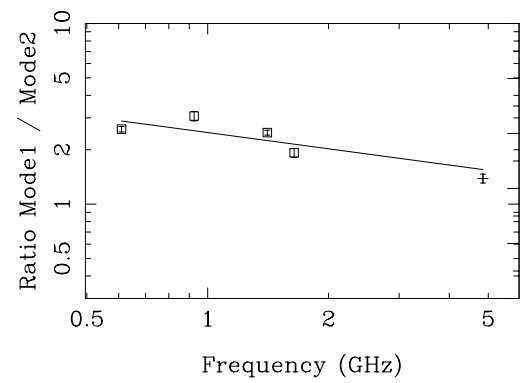

B1133+16 (right component)

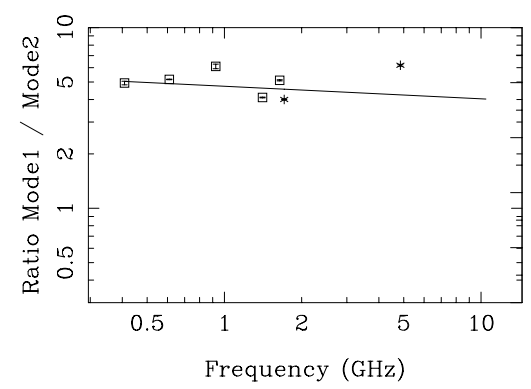

B1237+25 (right component)

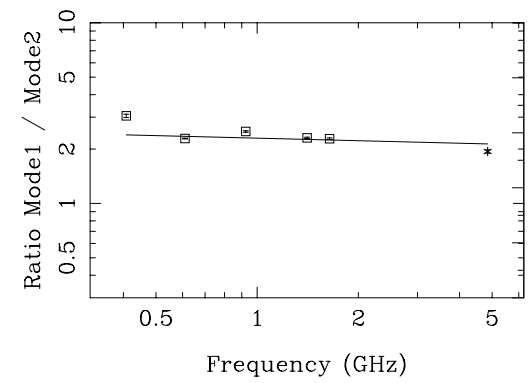

B1039-19 (both components)

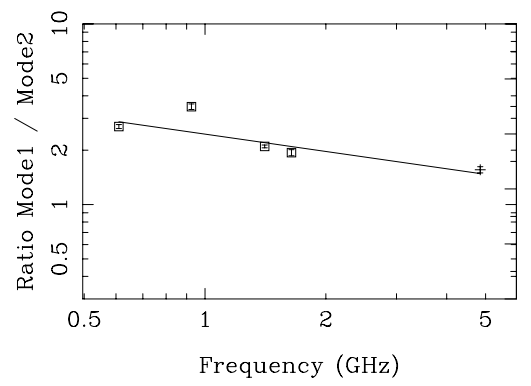

B1133+16 (both components)

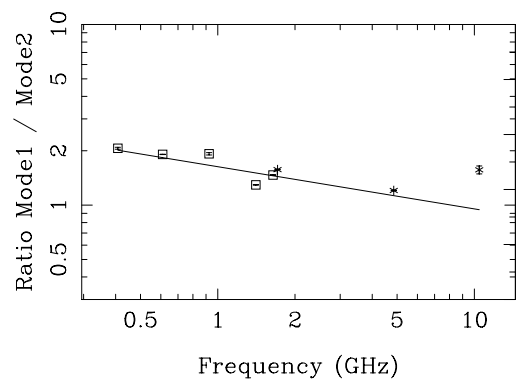

B1237+25 (both components)

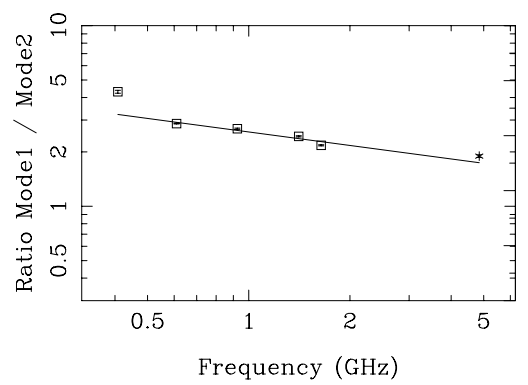

B1737-30

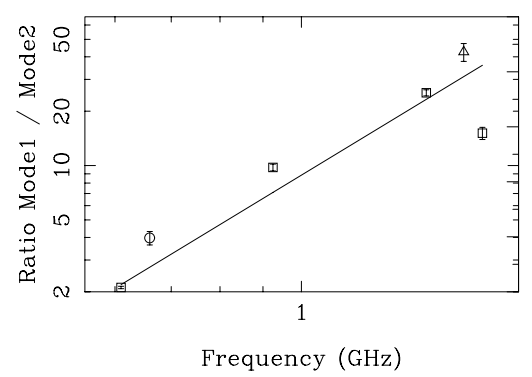

Fig. 23. See Fig. 22 for explanation. Note that for PSR B1737-30 the vertical axis is shifted with respect to the other plots. This does not affect the slope. 
J. M. Smits et al.: Frequency dependence of orthogonal polarisation modes in pulsars, Online Material p 22

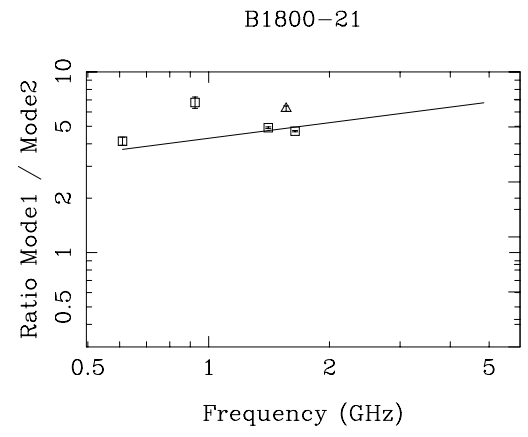

$\mathrm{B} 2016+28$

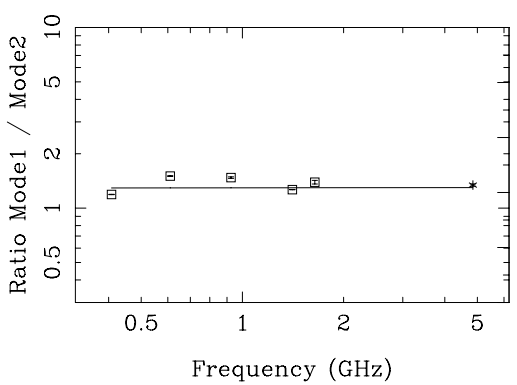

B2020+28 (right component)

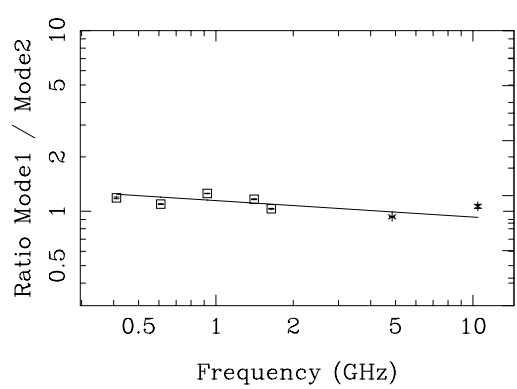

$\square \quad$ Gould \& Lyne (1998)

- $\quad$ von Hoensbroech et al. (1998)

$\times \quad$ von Hoensbroech \& Xilouris (1997)

$\bigcirc$ Guojun et al. (1995)

$\triangle \quad$ Wu et al. (1993)

. $\quad$ Seiradakis et al. (1995)

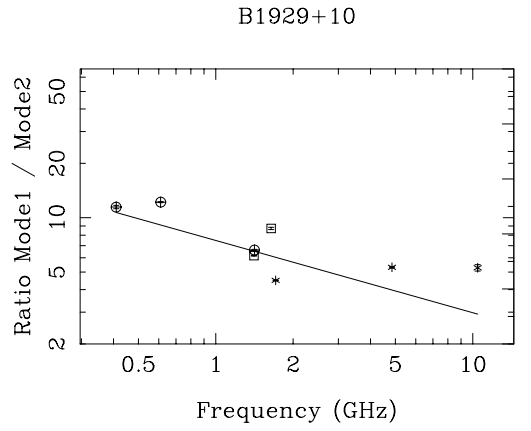

B2020+28 (both components)

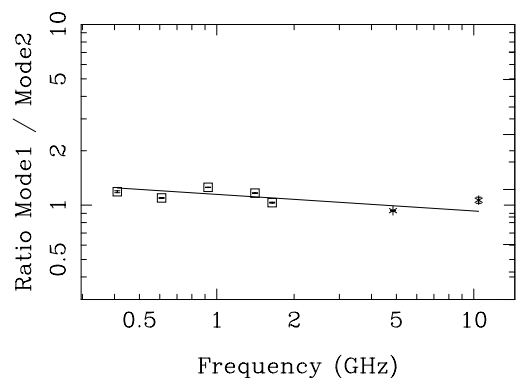

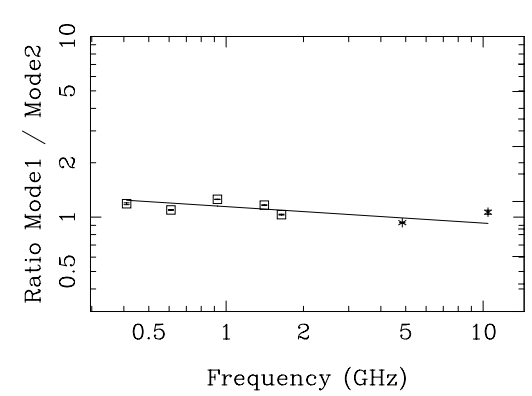

B1944+17

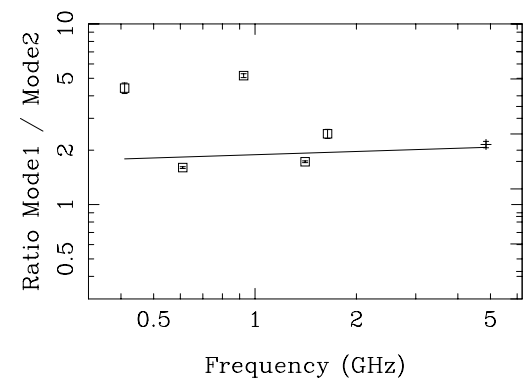

B2020+28 (left component)

Fig. 24. See Fig. 22 for explanation. Note that for PSR B1929+10 the vertical axis is shifted with respect to the other plots. This does not affect the slope. 